\title{
Probing the topological Anderson transition with quantum walks
}

\author{
Dmitry Bagrets $\odot,{ }^{1}$ Kun Woo Kim, ${ }^{1,2}$ Sonja Barkhofen, ${ }^{3}$ Syamsundar De $\odot,{ }^{3}$ Jan Sperling $\odot,{ }^{3}$ Christine Silberhorn, ${ }^{3}$ \\ Alexander Altland, ${ }^{1}$ and Tobias Micklitz ${ }^{4}$ \\ ${ }^{1}$ Institut für Theoretische Physik, Universität zu Köln, Zülpicher Straße 77, 50937 Köln, Germany \\ ${ }^{2}$ Department of Physics, Chung-Ang University, 06974 Seoul, Korea \\ ${ }^{3}$ Integrated Quantum Optics Group, Institute for Photonic Quantum Systems (PhoQS), Paderborn University, \\ Warburger Straße 100, 33098 Paderborn, Germany \\ ${ }^{4}$ Centro Brasileiro de Pesquisas Físicas, Rua Xavier Sigaud 150, 22290-180 Rio de Janeiro, Brazil
}

(Received 9 February 2021; accepted 4 May 2021; published 4 June 2021)

\begin{abstract}
We consider one-dimensional quantum walks in optical linear networks with synthetically introduced disorder and tunable system parameters allowing for the engineered realization of distinct topological phases. The option to directly monitor the walker's probability distribution makes this optical platform ideally suited for the experimental observation of the unique signatures of the one-dimensional topological Anderson transition. We analytically calculate the probability distribution describing the quantum critical walk in terms of a (timestaggered) spin polarization signal and propose a concrete experimental protocol for its measurement. Numerical simulations back the realizability of our blueprint with current date experimental hardware.
\end{abstract}

DOI: 10.1103/PhysRevResearch.3.023183

\section{INTRODUCTION}

Low-dimensional disordered quantum systems can escape the common fate of Anderson localization once topology comes into play, as witnessed at the integer quantum Hall plateau transitions $[1,2]$. The advent of topological insulators has brought a systematic understanding of topological Anderson insulators and their phase transitions [3]. The hallmark of an Anderson insulating phase with nontrivial topology (coined topological Anderson insulator) is the presence of topologically protected chiral edge states [4,5]. The Anderson localization transition by itself is characterized by the critical states which typically show unusual spectral- and wave-function statistics [6], as well as anomalous diffusive dynamics. From the single parameter scaling theory of localization one, e.g., expects a scaling $\left\langle\boldsymbol{q}^{2}(t)\right\rangle \sim t^{2 / d}$ of the mean square displacement at a conventional $d$-dimensional localization transition [7]. Topological localization transitions, on the other hand, follow a two-parameter scaling and the situation is more complex [8-10]. The controlled experimental study of a critical state at the Anderson localization transition presents an intriguing challenge. It has been first accomplished within a cold-atom realization of the quantum kicked rotor for the three-dimensional Anderson localization transition in the orthogonal class [11]. A corresponding study of a topological localization transition requires control over additional internal degrees of freedom [12].

Published by the American Physical Society under the terms of the Creative Commons Attribution 4.0 International license. Further distribution of this work must maintain attribution to the author(s) and the published article's title, journal citation, and DOI.
The physics of the topological Anderson localization transition is particularly intriguing in one-dimensional (1D) systems, where disorder is exceptionally efficient in inducing quantum localization on short length scales. Topological quantum criticality then reflects a competition between two powerful principles: strong localization versus the enforced change of an integer topological invariant. Topology trumps localization and forces long-range correlations across the system. In practical terms, this implies a divergent localization length and finite conduction. However, the reluctance of the system to conduct shows in an extremely (logarithmically) slow spreading of quantum states at criticality $[13,14]$, strikingly different to the diffusive dynamics conventionally observed at quantum phase transitions between disordered phases. In this paper, we connect the physics of 1D topological quantum criticality to the unique opportunities offered by quantum optics experimentation. We present a concrete and experimentally realistic blueprint for a tunable 1D quantum walk in which the unique signatures of topological quantum criticality show via a (time-staggered) spin polarization signal.

Quantum walks [15] have been implemented on various experimental platforms, such as photons [16-25], ions [26,27], atoms [28-30] and nuclear magnetic resonance [31]. A detailed introduction to experimental implementations of quantum walks can be found in Ref. [32]. Quantum walks allow for a large tunability of the system parameters and have been used experimentally to observe Anderson localization [23,25,33], dynamical localization [28], and topological effects [34-41]. Quantum walk systems thus open the perspective of a low-dimensional system in which disorder and nontrivial topology can be introduced in controlled manners. Direct experimental access to the probability distribution allows, moreover, for a full characterization of the walker's dynamics. 


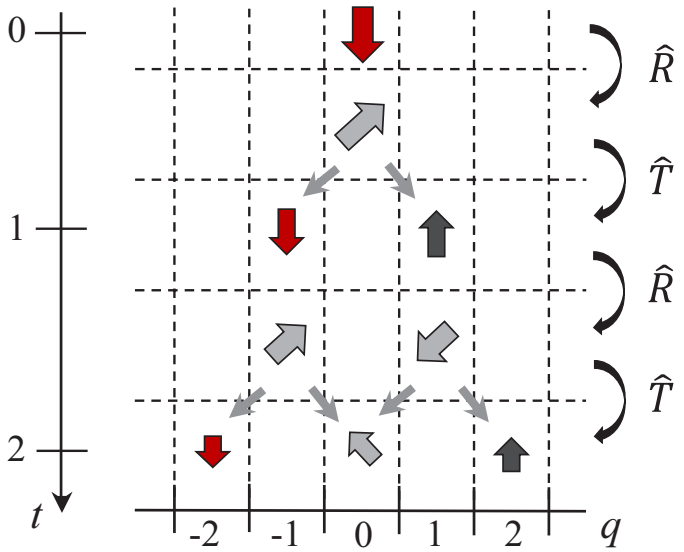

FIG. 1. A schematic evolution of a discrete-time quantum walk of a spin-1/2 particle over two steps in time. Alternating application of coin $\hat{R}$ and step $\hat{T}$ operators describe the dynamics on discrete lattice sites $q \in \mathbb{Z}$. In step two at position $q=0$, the first interference takes place.

A prototypical quantum walk is depicted in Fig. 1. It is generated by the single time-step evolution $\hat{U}=\hat{R} \hat{T}$, iteratively acting on a walker with a two-dimensional internal degree of freedom, referred to as spin in the following. Here $\hat{T}$ translates the particle on a discrete 1D lattice. Depending on its internal spin state, the walker propagates to the left or right and $\hat{R}$ is a rotation in spin-space. Using linear optical elements, discrete time quantum walks have been used to measure probability distributions of walkers exposed to tunable disorder and decoherence [33,42]. Specifically, conditions for ballistic, Anderson-localized, and classically diffusive dynamics were prepared and the corresponding walker's probability distributions [see also Eq. (9)] were observed. That is, the following scenarios apply: (i) $P_{\sigma^{\prime} \sigma}(t, q) \sim \delta(t-\sigma q) \delta_{\sigma \sigma^{\prime}}$ for a translational invariant quantum system (here $\sigma= \pm$ denotes the spin state), (ii) $P_{\sigma^{\prime} \sigma}(t, q) \sim \exp \left(-|q| / \xi_{\text {loc }}\right)$ for a disordered quantum system, and (iii) $P_{\sigma^{\prime} \sigma}(t, q) \sim \exp \left(-q^{2} / D t\right)$ for a disordered classical system, where $\xi_{\text {loc }}$ and $D$ are localization length and diffusion constant. In the photonic implementation, the internal spin states correspond to horizontal, $|H\rangle$, and vertical, $|V\rangle$, polarization directions, and disorder is controlled by local variations of polarization plates [33]. Rotations that do not explore all SU(2) angles independently leave symmetries, which can place the walk into one of the five nonstandard symmetry classes hosting topologically interesting phases [43-45].

In this paper, we explore a quantum walk operating at a topological Anderson localization transition. We derive the walker's critical probability distribution at the topological transition and find a (time-staggered) spin polarization as smoking-gun evidence for the critical dynamics. We indicate a protocol which allows for an observation of the discussed features within existing experimental platforms and compare results of our effective field theory approach to numerical simulations. The remainder of the paper is organized as follows. In Sec. II, we introduce a quantum walk with chiral symmetry that can be tuned to a quantum critical point separating two topologically different Anderson insulators. In Sec. III, we analyze the probability distribution of the critical walker and propose, in Sec. IV, an experimental protocol that allows one to study the predicted effect. We conclude in Sec. V with a discussion and an outlook. Several technical discussions are relegated to appendices.

\section{CHIRAL QUANTUM WALK}

We start our discussion with a general 1D quantum walk of a spin- $1 / 2$ particle encoded in the single time-step evolution [45]:

$$
\hat{U}(\vartheta, \varphi, \theta)=\hat{R}(\vartheta, \varphi, \theta) \hat{T} .
$$

The spin-dependent shift operator $\hat{T}$ here is diagonal in the $\hat{s}_{3}$ eigenbasis,

$$
\hat{T}=\sum_{q}(|q+1, \uparrow\rangle\langle\uparrow, q|+| q-1, \downarrow\rangle\langle\downarrow, q|),
$$

where $q \in \mathbb{Z}$ are the lattice sites with unit spacing, and spin states $\{|\uparrow\rangle,|\downarrow\rangle\}$ parametrize the walker's two-dimensional internal degrees of freedom, see also Fig. 1. Local coin rotations,

$$
\hat{R}=\sum_{q, \sigma \sigma^{\prime}}|q, \sigma\rangle R_{q}^{\sigma \sigma^{\prime}}\left\langle q, \sigma^{\prime}\right|,
$$

are conveniently parametrized by (site-dependent) Euler angles $R_{q}(\vartheta, \varphi, \theta)=\exp \left(\frac{i}{2} \vartheta_{q} \sigma_{1}\right) \exp \left(i \varphi_{q} \sigma_{3}\right) \exp \left(\frac{i}{2} \theta_{q} \sigma_{1}\right)$, where Pauli matrices $\sigma_{i}$ operate in spin space. Employing a symmetrized time-splitting, we can write

$$
\hat{U}(\vartheta, \varphi, \theta)=R_{z}\left(\frac{\hat{\varphi}}{2}\right) R_{x}\left(\frac{\hat{\theta}}{2}\right) \hat{T} R_{x}\left(\frac{\hat{\vartheta}}{2}\right) R_{z}\left(\frac{\hat{\varphi}}{2}\right),
$$

with $\hat{\vartheta}, \hat{\varphi}, \hat{\theta}$ being site-diagonal matrices of angles and $R_{i}(\hat{\alpha})=\exp \left(i \hat{\alpha} \sigma_{i}\right)$ defines a rotation along the $i$ th direction. From Eq. (4), one readily verifies that quantum walks subject to the constraint $\hat{\theta}=\hat{\vartheta}$ exhibit a chiral symmetry [46]:

$$
\sigma_{2} \hat{U} \sigma_{2}=\hat{U}^{\dagger} .
$$

That is, the latter are members of the chiral symmetry class AIII, which may host $\mathbb{Z} \times \mathbb{Z}$ topological insulating phases for 1D quantum walks [44]. Throughout this paper, we only focus on walks with $\hat{\theta}=\hat{\vartheta}$, i.e., with the chiral symmetry denoted in Eq. (5).

Chiral symmetry of the time-evolution operator reflects in a spectrum which is symmetric around zero in the $2 \pi$-periodic Brillouin zone of quasienergies $\epsilon_{p}^{ \pm}= \pm \epsilon_{p}$. For spatially constant rotations, Floquet eigenstates are plane waves and the two energy bands are defined by the relation

$$
\cos \left(\epsilon_{p}(\theta, \varphi)\right)=\cos (\varphi+p) \cos ^{2}\left(\frac{\theta}{2}\right)-\cos (\varphi-p) \sin ^{2}\left(\frac{\theta}{2}\right),
$$

with $p$ being the momentum of the plane wave. Finite angles $\varphi$ and $\theta$ shift the momentum and tune the bandwidth of Bloch bands; see Fig. 2. Specifically, linearly dispersing bands that extend over the entire Brillouin zone exist at values $\theta=0, \pi$, with

$$
\epsilon_{p}(\theta, \varphi)=\theta+p+e^{i \theta} \varphi
$$




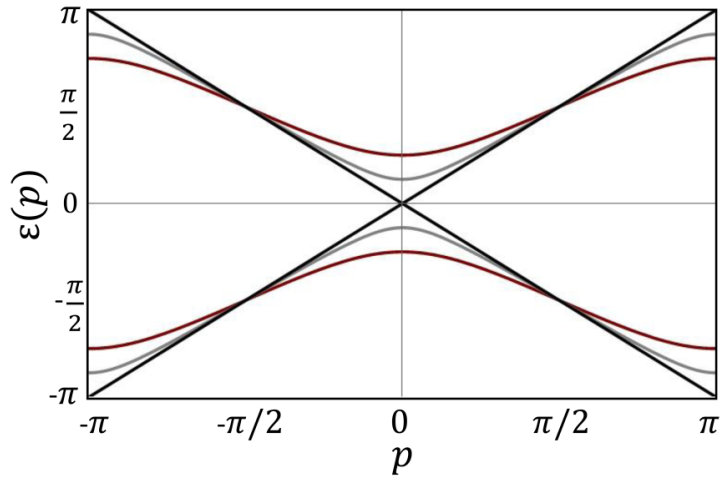

FIG. 2. Dispersion relation $\epsilon(p)$ of Floquet bands shown for angles $\varphi=0$ and $\theta=0$ (black line), $\pi / 8$ (grey), and $\pi / 4$ (red).

and $\varphi= \pm \frac{\pi}{2}$, with

$$
\epsilon_{p}(\theta, \varphi)=\frac{\pi}{2} \pm \operatorname{sgn}(\varphi) p .
$$

For any other values of the angles, the spectrum is gapped around $\epsilon=0$ and $\pi$. Disorder can be introduced in a controlled manner by randomizing angles. Assuming short-range site-to-site correlations, rotations are then characterized by average angles $\bar{\theta}, \bar{\varphi}$, and their deviations $\gamma_{\theta}, \gamma_{\varphi}$, which we assume to be identical for all lattice sites. In one dimension, even weak disorder $\gamma_{\theta, \varphi} \ll 1$ strongly affects the dynamics of the walker, turning its ballistic propagation into exponential Anderson localization on the scale of the mean-free path [47], which is set by the spatial scale on which the random rotation angles fluctuate. The presence of the chiral symmetry, Eq. (5), on the other hand, allows the quantum walker to escape the common fate of Anderson localization. This happens when the system is fine-tuned to the quantum critical point, separating two topologically different Anderson insulating phases, as we discuss next.

\section{CRITICAL DISTRIBUTION}

To elaborate on the last mentioned point, we consider the probability distribution,

$$
P_{\sigma^{\prime} \sigma}(t, q)=\left\langle\left|\left\langle q, \sigma^{\prime}\left|\hat{U}^{t}\right| 0, \sigma\right\rangle\right|^{2}\right\rangle_{\theta, \varphi},
$$

for a walker who is initially in eigenstate $|\sigma\rangle=|\leftarrow\rangle,|\rightarrow\rangle$ of the chiral operator $\hat{\sigma}_{2}$. This distribution yields the probability of the walker to be found after $t$ time steps at a distance $q$ in eigenstate $\left|\sigma^{\prime}\right\rangle$. Here and in the following, $\langle\ldots\rangle_{\theta, \varphi}$ denotes averages over distributions of angles. Since particles conserve their (quasi)energies, it is convenient to Fourier transform Eq. (9) to a spectral representation:

$$
P_{\sigma^{\prime} \sigma}(\omega, q)=\int d \epsilon\left\langle\left\langle q, \sigma^{\prime}\left|\hat{G}_{\epsilon+\frac{\omega}{2}}^{R}\right| 0, \sigma\right\rangle\left\langle 0, \sigma\left|\hat{G}_{\epsilon-\frac{\omega}{2}}^{A}\right| q, \sigma^{\prime}\right\rangle\right\rangle_{\theta, \varphi} .
$$

Here we introduced the retarded (particle) and advanced (hole) propagators $\hat{G}_{\epsilon}^{R}=\left[1-e^{i \epsilon-0} \hat{U}\right]^{-1}$ and $\hat{G}_{\epsilon}^{A}=\left[\hat{G}_{\epsilon}^{R}\right]^{\dagger}$, respectively.

The chiral symmetry relates particle and hole dynamics for states in the vicinity of particle-hole symmetric points $\epsilon \simeq 0, \pi$ in the $2 \pi$-periodic spectrum. More specifically, the chiral symmetry Eq. (5) translates into the relation

$$
\hat{G}_{-\epsilon}^{A}=\sigma_{2} \hat{G}_{\epsilon}^{R} \sigma_{2},
$$

indicating that the dynamics of particles and holes at a fixed energy is only related for $\epsilon \simeq-\epsilon$. That is, for states in the vicinity of particle-hole symmetric points, $\epsilon \simeq 0, \pi$.

To account for the (breaking of) symmetry between particle and hole propagators in different ranges of the quasienergy spectrum, we change to an energy representation of Eq. (9) and separate Fourier components into two contributions [10]:

$$
P_{\sigma^{\prime} \sigma}(\omega, q) \simeq P^{\mathrm{reg}}(\omega, q)+P_{\sigma^{\prime} \sigma}^{\mathrm{chiral}}(\omega, q) .
$$

Herein, the first (spin-independent) contribution results from single-particle states, with $|\epsilon|,|\epsilon-\pi| \gtrsim \omega$, for which energy detuning is large enough to break the chiral symmetry between propagators that compose the probability distribution as $P \sim G^{R} G^{A}$. Consequently, the probability distribution for states breaking the chiral symmetry is (on long time and length scales) identical to that of conventional Anderson insulators. That is, upon Fourier transform, the first contribution is given by a static probability distribution:

$$
P^{\text {reg }}(t, q) \sim \theta(t) e^{-|q| / \xi_{\text {loc }}} .
$$

By contrast, the second contribution results from states with quasienergies $|\epsilon|,|\epsilon-\pi| \lesssim|\omega|$, for which both propagators are related by chiral symmetry. That is,

$$
P_{\sigma^{\prime} \sigma}^{\text {chiral }}(\omega, q) \simeq|\omega|\left\langle\left\langle q, \sigma\left|\hat{G}_{\frac{\omega}{2}}^{R}\right| 0, \sigma\right\rangle\left\langle 0, \sigma\left|\hat{G}_{-\frac{\omega}{2}}^{A}\right| \sigma, q\right\rangle\right\rangle_{\theta, \varphi}
$$

encodes the critical dynamics of the walker, and a nontrivial time resolved behavior can be expected. We next apply fieldtheory methods to identify quantum critical points of the chiral walk and calculate the critical distribution in Eq. (14).

\section{A. Sinai diffusion}

Following the standard approach to disordered systems [8,48-52], we describe the physics of the critical states around $\epsilon \simeq 0, \pi$ by a Ginzburg-Landau-type effective theory. More specifically, we derive in Appendix A a nonlinear $\sigma$-model action which encodes the full quantum dynamics of soft diffusion modes and interference processes which eventually drive strong Anderson localization. What changes this conventional behavior in our case is a topological contribution to the effective action.

The $\sigma$ model is parametrized by the frequency $\omega$ (which, however, does not flow in a renormalization group sense), and two coupling constants, viz. the conductance $\mathfrak{g}$ and topological angle $\chi$, see Eq. (A25). In a conventional disordered system, $\mathfrak{g}$ follows a single parameter scaling [53], which in $1 \mathrm{D}$ predicts a single, Anderson insulating phase. The angle $\chi$, on the other hand, allows for a characterization of topologically different Anderson insulating phases. This opens the possibility to escape Anderson localization when fine-tuned to specific, critical values, separating two topologically distinct Anderson insulators.

The bare topological angle for the chiral quantum walk reads (see Appendix A for details)

$$
\bar{\chi}^{\epsilon}=\frac{1}{2}\left(1-e^{i \epsilon}\langle\sin (\theta) \cos (\varphi)\rangle_{\theta, \varphi}\right),
$$




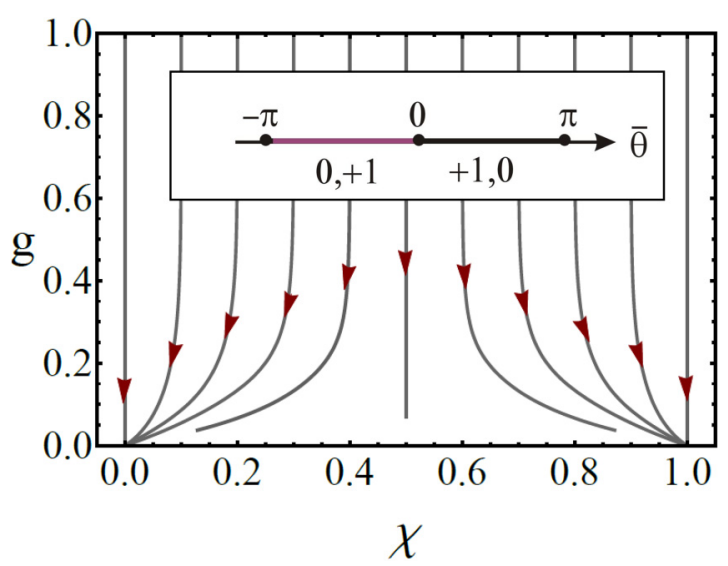

FIG. 3. Two-parameter flow of conductance $g(L)$ and average topological index $\chi(L)$ for class AIII nonlinear $\sigma$-model with bare values $\mathfrak{g}(1) \sim 1$ and $\chi(1)=\bar{\chi}$. Inset: Phase diagram of the quantum walk. Assuming $\bar{\varphi} \neq \pm \pi / 2$, the system is at criticality provided $\bar{\theta}=0$ or $\pi$. Away from criticality, the pair of topological indices $\left(\chi^{0}, \chi^{\pi}\right)$ flow to either $(0,1)$ or $(1,0)$, which defines two distinct Anderson localized topological phases. For $\bar{\varphi}= \pm \pi / 2$, the system is always critical.

with $\epsilon=0, \pi$ indicating the critical states described by the effective action. The presence of two coupling constants in the $\sigma$-model action-the conductance and topological angle $[10,54,55]$ - leads to a two-parameter flow and resulting phase diagram shown in Fig. 3 [10]. For generic bare values, the average topological angle flows to the closest integer value $\chi=$ 0 and 1 , characterizing the two Anderson insulating phases realized by the chiral quantum walk, while $\chi=1 / 2$ defines the critical fixed-point line. For general $\bar{\varphi}$, a critical line corresponds to $\bar{\theta}=0, \pm \pi$. The same configuration of angles in the clean limit gives rise to gapless linearly dispersing bands, as expected from analogy to the corresponding Hamiltonian system. For generic $\bar{\theta}$, on the other hand, criticality is achieved at $\bar{\varphi}= \pm \pi / 2$. Finally, we remark that, in the strong disorder limit where angels are randomly drawn from the entire unit circle, $\gamma_{\theta, \varphi}=2 \pi$, the quantum walk is always critical. The same effective action also describes disordered quantum wires with chiral symmetry and bare localization length $\xi_{\text {loc }}=1 / 2$ [10]. Criticality in the strong disorder limit is, however, a peculiarity of the Floquet system.

Concentrating then on the vicinity of a critical point, we can calculate the walker's critical distribution Eq. (14). The rather technical calculation is detailed in Appendix B and indicates the scaling form

$$
P_{\sigma^{\prime} \sigma}^{\text {chiral }}=\mathcal{N}(t) \mathcal{F}_{\sigma^{\prime} \sigma}\left(|q| \xi_{t}^{-1}\right), \quad \xi_{t} \equiv \frac{2}{\pi^{2}} \ln ^{2} t,
$$

with a time-dependent normalization factor $\mathcal{N}(t) \propto \ln ^{-5} t$ and the explicit form of $\mathcal{F}(x)$ to be given given below [56]. Notice that this implies that $\sum_{q, \sigma^{\prime} \sigma} P_{\sigma^{\prime} \sigma}^{\text {chira }}(t, q)=1 /\left(4 \ln ^{2} t\right)$, as shown in Appendix B. The scaling of $\xi_{t}$ implies anomalously retarded Sinai diffusion of critical states, characterized by a mean displacement [57-59]:

$$
\langle|q|\rangle \sim \ln ^{2} t .
$$

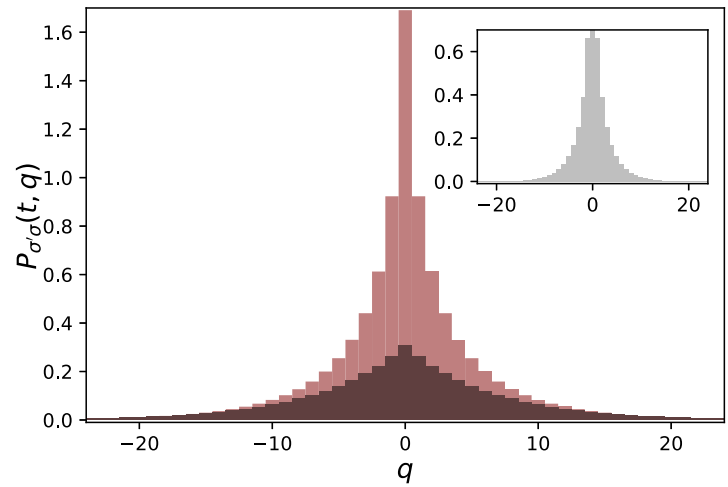

FIG. 4. Walker's critical probability distribution $P_{\sigma^{\prime} \sigma}^{\text {chiral }}(t, q)$, Eq. (18), for $t=10$. Spin-configurations $\left(\sigma^{\prime}, \sigma\right)$ of final and initial states are aligned $(\rightarrow, \rightarrow)$ (light red) and anti-aligned $(\leftarrow, \rightarrow)$ (dark red), and distributions are normalized by the average return probability per spin. Inset: Spin polarization $\Delta P(t, q)$ (peak at origin is not fully shown).

Another feature of the critical distribution is the dependence on spin orientations $\sigma \sigma^{\prime}= \pm 1$ with reference to the chiral symmetry $\sigma_{2}$. This can be seen from the scaling functions in the long-time and distance limits, $t, q \gg 1$ :

$$
P_{\sigma^{\prime} \sigma}^{\text {chiral }}(t, q) \propto \frac{1}{\ln ^{5} t} \sum_{n=1}^{\infty}\left(\sigma \sigma^{\prime}\right)^{(n+1)} n^{2} e^{-n^{2}|q| / \xi_{t}} .
$$

Focusing on the tails $|q| \gg \xi_{t}$, one finds from Eq. (18) the exponential profiles

$$
\mathcal{F}_{ \pm \sigma, \sigma}(x)=e^{-x} \pm 4 e^{-4 x}+\ldots,
$$

where the leading spin-independent contribution reminds us of conventional Anderson insulators. The directly following terms indicate, however, dependence of the critical distribution on the spin orientation of the final state $\pm \sigma$, with interesting implications, being discussed in the next subsection. The full distributions [cf. Eq. (18)] are shown in Fig. 4, and we refer the interested reader to Appendix B for a more detailed analytical expression. We next discuss how the characteristic features of the critical distribution, viz. (i) slowly increasing width $\xi_{t}$ in time and (ii) dependence on spinorientation with respect to the basis of the chiral symmetry operator, can be observed in experiments.

\section{B. Time-staggered spin polarization}

Sinai diffusion has previously been predicted for disordered 1D systems with particle-hole symmetry $[13,14]$ and our above result for a system with chiral symmetry indicates that it is a universal dynamical feature at 1D topological Anderson localization transitions. Arguably, observation of the weak logarithmic time dependence presents an experimentally intriguing challenge [60]. Recalling, moreover, the contribution from noncritical states to the total probability distribution implies that Sinai diffusion is generally masked by conventionally Anderson localization. Complicating this matter even further, the number of critical states resolved in time $t$ reduces as $|\omega| \sim 1 / t$. This generates additional time dependencies in the distributions of noncritical and critical 
states, as summarized in the normalization $\mathcal{N}(t)$ of Eq. (16). The optical linear network realization of a quantum walk discussed in the Introduction, on the other hand, allows for a direct observation of spin-resolved probability distributions. This opens an interesting opportunity to observe the second feature, i.e., the peculiar spin dependence of the critical walk. Specifically, this suggests to measure the difference

$$
\Delta P(t, q) \equiv P_{\rightarrow \rightarrow}^{\text {chiral }}(t, q)-P_{\leftarrow}^{\text {chiral }}(t, q),
$$

which only depends on the critical contribution to the total probability. A finite spin polarization of the critical walker may be viewed as a precursor of spin polarized boundary states emerging in the topologically nontrivial phase [61-63].

The $q$ dependence of the difference $\Delta P(t, q)$ is shown in the inset of Fig. 4. The corresponding long time probability distributions for noncritical states and conventional Anderson insulators (with spin orbit interaction) do not keep the memory of the initial spin configuration. The observation of $\Delta P(t, q)$ would thus provide clear evidence for the critical walk at an Anderson localization transition.

Further smoking-gun evidence for the critical distribution is then obtained from an additional symmetry of the Floquet operator not discussed so far. The discrete lattice structure motivates the introduction of the sublattice operator [64]

$$
\hat{S} \equiv \sum_{q}|q\rangle(-1)^{q}\langle q|
$$

anticommuting with the Floquet operator $\hat{U}$. From Eq. (21), one can construct a chiral-sublattice operator

$$
\hat{\mathcal{C}}_{\mathrm{sl}} \equiv \sigma_{2} \otimes \hat{S}
$$

satisfying $\hat{\mathcal{C}}_{\mathrm{sl}} i \hat{U} \hat{\mathcal{C}}_{\mathrm{sl}}=(i \hat{U})^{\dagger}$ and consequently resulting in

$$
\hat{G}_{\epsilon_{0}-\epsilon}^{A}=\hat{\mathcal{C}}_{\mathrm{sl}} \hat{G}_{\epsilon_{0}+\epsilon}^{R} \hat{\mathcal{C}}_{\mathrm{sl}},
$$

whenever $\epsilon_{0}= \pm \frac{\pi}{2}$. That is, $\hat{\mathcal{C}}_{\mathrm{sl}}$ is an additional chiral symmetry that applies to critical states $\epsilon \simeq \pm \frac{\pi}{2}$. This symmetry is also visible in the density of states (DoS), as we show in Appendix C. Interestingly, $\hat{\mathcal{C}}_{\mathrm{sl}}$ has different implications for time evolution when extending over an even or odd number of time steps $t$. We show in Appendix D that, for critical states induced by the chiral-sublattice symmetry $\hat{\mathcal{C}}_{\mathrm{sl}}$, the spin polarization alternates in between time steps; that is,

$$
\Delta P(t, q)=(-1)^{t}|\Delta P(t, q)|
$$

holds true. The two main observations leading to Eq. (24) are the following (for a more rigorous explanation, see Appendix D): (i) The Floquet operator induces transitions between subspaces of opposite site parities. That is, walkers positioned at an even site propagate in the following time step to an odd site and vice versa. (ii) Eigenstates of $\hat{\mathcal{C}}_{\text {sl }}$ have alternating spin polarization on even and odd sites; e.g., $|q, \rightarrow\rangle$ are eigenstates of $\hat{\mathcal{C}}_{\mathrm{sl}}$ with eigenvalues $(-1)^{q}$ and analogously for $|q, \leftarrow\rangle$. Combining both observations, we notice that walkers propagating for an even number of time steps have spins aligned if their initial and final states are eigenstates of $\hat{\mathcal{C}}_{\mathrm{sl}}$ to the same eigenvalue. (The same applies for the chiral operator $\sigma_{2}$.) In contrast, for an odd number of time steps, the walker has spins antialigned if initial and final states are eigenstates of $\hat{\mathcal{C}}_{\mathrm{sl}}$ to the same eigenvalue. This difference simply follows

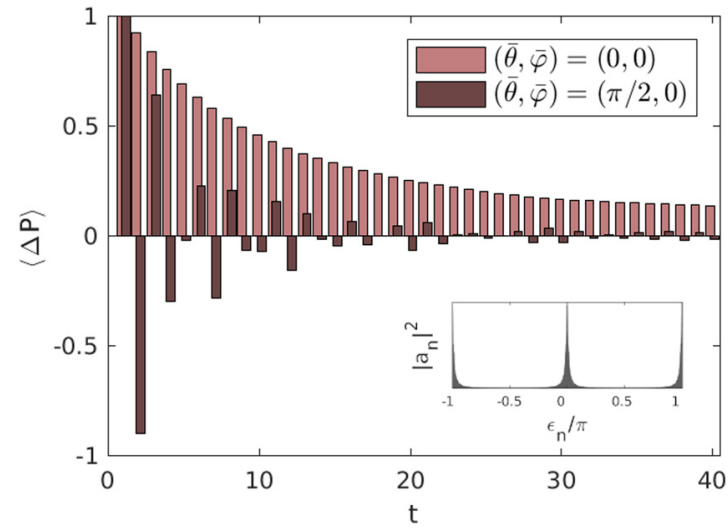

FIG. 5. Spin polarization $\Delta P(t)$ as a function of time steps $t$ for the initial states $\left|\psi_{M}^{0}\right\rangle$ with $M=10^{2}$ and different choices of mean angles $(\bar{\theta}, \bar{\varphi}) . \Delta P(t)$ remains finite for a large number, $t \sim O\left(10^{2}\right)$, of time steps (light red) if the walker probes critical states $\epsilon \simeq 0$ corresponding to $(\bar{\theta}, \bar{\varphi})=(0,0)$ (cf. a spectral decomposition of the initial state as shown in the inset, with $\left.a_{n}=\left|\left\langle\epsilon_{n} \mid \psi_{M}^{0}\right\rangle\right|^{2}\right)$. On other hand, $\Delta P(t)$ is scrambled (dark red) if noncritical states at $(\bar{\theta}, \bar{\varphi})=(\pi / 2,0)$ are probed.

from the observation that, for an odd number of time steps, the walk starts and ends in opposite parity sectors. Finally, we also remark that the probability $P_{\sigma^{\prime} \sigma}$ has to be read as the transition within $\left(\sigma^{\prime}=\sigma\right)$ or between $\left(\sigma^{\prime}=-\sigma\right)$ eigenspaces of the chiral operator. Combining the above, it follows that for odd numbers of time steps, spin polarization reverses its sign. We thus conclude that, for critical states induced by the chiral-sublattice symmetry $\hat{\mathcal{C}}_{\mathrm{sl}}$, the spin polarization alternates in between time steps, as indicated in Eq. (24).

\section{EXPERIMENTAL PROPOSAL}

We now devise an experimental protocol which allows us to observe the discussed characteristic features of the quantum critical walk.

\section{A. Experimental protocols}

Quantum walks are typically initialized at a localised position and thus involve eigenmodes from the entire quasienergy domain. The experimental challenge then is to restrict the dynamics to states that approximately preserve chiral symmetry. As discussed in the previous section, the Floquet evolution operator of the quantum walk induces the transitions between the sites of different parity only. We thus suggest to prepare an initial state of the single photon as a coherent superposition described by

$$
\left|\psi_{M}^{p_{0}}\right\rangle=\frac{1}{\sqrt{M+1}} \sum_{|q| \leqslant M / 2}|(2 q)\rangle \otimes|\rightarrow\rangle e^{2 i q p_{0}},
$$

which occupies $(M+1)$ even lattice sites, where $M \gg 1$ can be used to enhance the population of states at energies $\pm \epsilon_{p_{0}}$ (see also below discussion of initial states in Figs. 5 and 6).

Alternatively, one can make use of the equivalence of coherent light and a single quantum particle when propagating in a linear optical network and directly apply a train of coherent laser pulses. With $\left|\psi_{M}^{p_{0}}\right\rangle$ as the delocalized initial state, time 


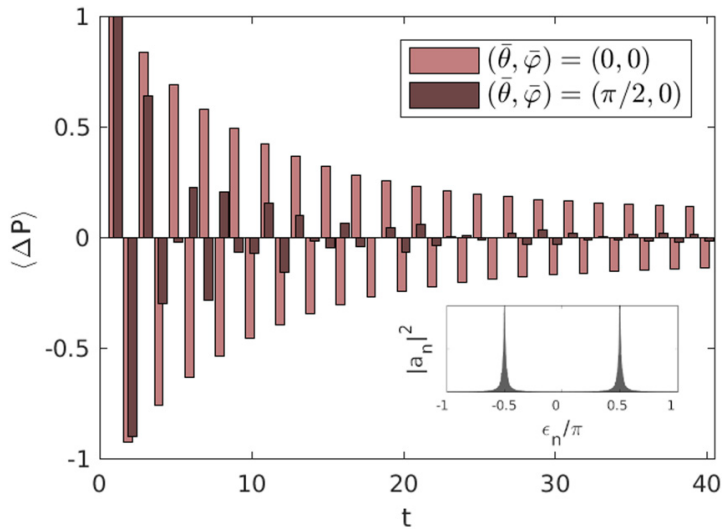

FIG. 6. The spin polarization $\Delta P(t)$ shows the predicted timestaggered behavior with a long-lived finite amplitude for the initial state $\left|\psi_{M}^{\pi / 2}\right\rangle$ with $M=10^{2}$ which is chosen to filter critical energies $\epsilon \simeq \pm \frac{\pi}{2}$ related to the chiral-sublattice symmetry $\hat{C}_{\mathrm{sl}}$. The criticality implies $(\bar{\theta}, \bar{\varphi})=(0,0)$ (light red). Similar to Fig. 5, $\Delta P(t)$ is scrambled when noncritical states at $(\bar{\theta}, \bar{\varphi})=(\pi / 2,0)$ are probed (dark red).

dependence of the localization length $\xi_{t}$ cannot be captured but the spatially integrated spin-polarization $\Delta P(t)$ can serve as a key measure for critical phases. Specifically, we define the latter as

$$
\Delta P(t) \equiv \sum_{q} \Delta P_{\psi}(t, q),
$$

where $\Delta P_{\psi}(t, q) \equiv P_{\rightarrow \psi}(t, q)-P_{\leftarrow \psi}(t, q)$ and the spindependent local probabilities are

$$
P_{\sigma \psi}(t, q) \equiv\left\langle\left|\left\langle q, \sigma\left|\hat{U}^{t}\right| \psi_{M}^{p_{0}}\right\rangle\right|^{2}\right\rangle_{\theta, \varphi} .
$$

Figures 5 and 6 show our numerical simulations for the spin polarization $\Delta P(t)$ using the initial state from Eq. (25) for $M=10^{2}$. We here did not assume periodic boundary conditions, i.e., the signal could propagate without restrictions to the left and right. In these plots, light red histograms simulate the critical walk at the topological Anderson localization transition, corresponding to critical energies $\epsilon \simeq 0, \pi$ (Fig. 5) and $\epsilon \simeq \pi / 2$ (Fig. 6), respectively. It can be clearly seen that a finite (spin-staggered) polarization is maintained up to over $t=40$ time steps, which is in the reach of current experiments, indeed [65-67]. By contrast, the dark red curve is a simulation of the quantum walk in a conventional Anderson insulating phase (see also phase diagram shown in inset of Fig. 3). In this case, the spin polarization of the initial state $\left|\psi_{M}^{p_{0}}\right\rangle$ quickly scrambles and decays. In all simulations, static uncorrelated angles $\theta_{q}$ and $\varphi_{q}$ were randomly drawn from intervals $(\bar{\theta}-\delta, \bar{\theta}+\delta)$ and $(\bar{\varphi}-\delta, \bar{\varphi}+\delta)$ of size $2 \delta=\pi / 4$, with $\bar{\theta}$ and $\bar{\varphi}$ referring to their mean values, and we performed ensemble average over $5 \times 10^{3}$ realizations. Overall, we find clear evidence of the discussed features in different variants of the suggested protocol, starting at a number of $t \sim O(10)$ time steps. As we have also checked, the results demonstrated in Figs. 5 and 6 remain qualitatively unchanged provided only one angle, $\varphi_{q}$, is random but $\theta_{q}=\bar{\theta}$ does not fluctuate, which potentially is easier to realize in practice as discussed in the next subsection.

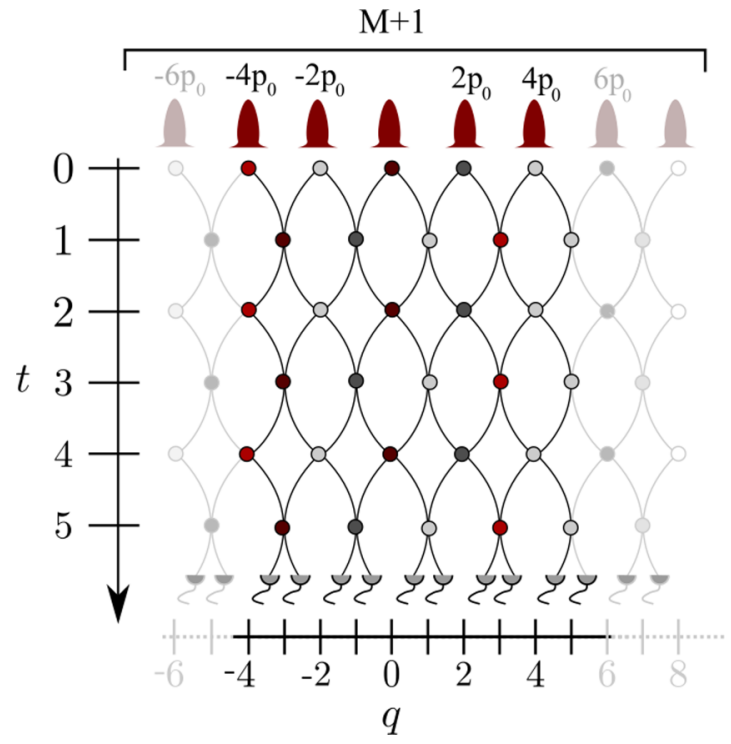

FIG. 7. A prototype of a linear optical network to realize a quantum critical walk discussed in detail in the main text. A train of $M+1$ coherent pulses with a fixed relative phase difference, $2 p_{0}$, between adjacent pulses enters neighboring ports of the network. Each vertex illustrated the coupling of two spatial modes and is implemented by a series of waveplates and a polarizing beam splitters, realizing the operators according to Eq. (4). The different colors of the vertices denote the (static) randomness in the phase $\varphi_{q}$. Eventually, the distribution for each disorder realization is measured polarization resolved in the circular basis.

\section{B. Experimental setup}

A schematic drawing of such a quantum critical walk is shown in Fig. 7. Pulses with a fixed phase relation are entering neighboring input modes of the network. Over the course of the evolution, they undergo polarization rotations with particular angles (indicated by the different colors of vertices) and a polarization dependent routing. Finally, the detectors resolve internal (i.e., polarization) as well as an external degree of freedom for extracting spin-resolved probability distributions. Ensemble averages over a few thousand realizations of disorder are necessary since a single realization only shows very little signatures of the critical states because of the impact of all the noncritical states. Only through this averaging procedure can the staggering behavior of the critical states becomes visible and be reliably extracted.

In addition to the precise control of all local coin rotations and the easy reconfigurability of the experiment to program the high number of realizations, the generation of the delocalized input state is one of the main experimental challenges. When considering a spatial implementation of the quantum walk network [19,68,69], a spatial-multiplexing techniques, as in Ref. [70], can be adopted to produce the input state to be fed into the network ports. Analogously, time-multiplexing networks $[20,71]$ can be adapted in the (temporal) position spacing to be directly compatible with the pulse train produced by a coherent cavity laser source. For the advanced control of the phase between the pulses, we envision the usage of directly modulated light source [72]. Alternatively, an external time-multiplexing loop, as suggested for driven 
quantum walks in Ref. [73] and which controls timings and phases of the initial pulse train, can be connected to the setup. Standard optical wave plates take care of the desired circular input polarization, resulting in the state $\left|\psi_{M}^{p_{0}}\right\rangle$-i.e., a state in the form of Eq. (25).

The nonrandom rotations $R_{x}(\bar{\theta} / 2)$ and the random rotation $R_{z}\left(\bar{\varphi}_{q} / 2\right)$, forming the coin operator, are implemented by (spatially varying) half (HWP) and quarter waveplates (QWPs), being positioned independently at each node of the network. In time-multiplexing networks, fast switching electro-optic modulators can be utilized to introduce the random phases $\varphi_{q}[21,33]$ in a controlled fashion. Since the evolution in the network typically takes place in horizontal and vertical polarization, the measurement basis has to be rotated again to circular states, such as by using QWPs at $45^{\circ}$ angle in front of the detectors. Crucially, to extract the spin polarization, $\Delta P(t)$, both polarization modes must be measured separately for every step. In Appendix E, we provide estimates for time and spacial scales which validate a feasibility of our proposal within existing experimental techniques.

\section{DISCUSSION}

We have studied the 1D quantum walk of a spin-1/2 particle with chiral symmetry and tunable disorder. The quantum walk allows us to realize topologically different Anderson insulating phases and can be tuned to a quantum critical point separating two such phases. Building on a low energy effective field theory, we have derived the walker's phase-diagram as a function of the average values of the coin operators rotation angles and their variances. Notice that in a periodically driven system, all eigenmodes in the quasienergy domain typically contribute to physical responses. In the presence of delocalized bulk modes, Floquet systems therefore cannot be called topological insulators (regardless of the presence of topological boundary modes and a finite gap in the quasienergy spectrum). This is different in the presence of disordered, where quantum interference drives the system into Anderson localized phases (as summarized in the twoparameter flow shown Fig. 3). We found that the critical point between two topologically distinct Anderson insulators is characterized by the spin-dependent probability distribution.

The critical dynamics reflects the competition between strong localization in a $1 \mathrm{D}$ system on the scale of the mean-free path and the formation of a nontrivial topological invariant which forces long range correlation through the system. We calculated the critical probability distribution of the walker and verified that the powerful, opposing strong localization in 1D manifests in extremely slow critical dynamics, that is, in Sinai diffusion as previously also found for quasi-1D disordered topological superconductors [14]. We identified a (time-staggered) spin polarization as a promising observable signature witnessing the quantum critical correlations. More specifically, we noticed that the walker's critical distribution keeps memory of the initial spin configuration when prepared in an eigenstate of the chiral operator. Moreover, we noted that the combination of chiral hopping on a discrete lattice and the chiral symmetry leads to a second chiral lattice symmetry. The spin polarization then becomes staggered in time when critical states protected by this second symmetry are probed.
The underlying mechanism suggests that, quite generally, in systems with chiral hopping on a discrete lattice, one may expect time staggering of observables which are sensitive to the eigenvalues of the chiral symmetry operator.

Taking advantage of the versatile opportunities offered by optics, we have proposed a protocol that should allow for the observation of the spin polarization within an existing experimental setup. One experimental challenge is to minimize contributions from uncritical states that suffer from conventional Anderson localization and which may mask the spin polarization. We proposed to filter critical states in the quasienergy spectrum by preparing the walker initially in a spatially extended state. We confirmed the viability of our proposal by numerically simulations of the protocol for experimentally realistic system parameters, and verified a strong suppression of the spin polarization by either activating uncritical states, breaking chiral symmetry, or introducing dephasing. For an experimental platform we, e.g., indicate an optical linear network similar to that used in Ref. [33]. The preparation of an extended initial state with a stable fixed phase relation may still be challenging. We are, however, optimistic that some variant of the protocol is in experimental reach in one of the discussed platforms. The experimental observation of the time-staggered spin polarization would provide intriguing evidence for the quantum critical dynamics manifesting as a competition of strong localization and nontrivial topology in disordered quantum systems.

\section{ACKNOWLEDGMENTS}

We wish to thank Benjamin Brecht for discussions. T.M. acknowledges financial support by Brazilian agencies $\mathrm{CNPq}$ and FAPERJ. A.A., D.B., and K.W.K. were funded by the Deutsche Forschungsgemeinschaft (DFG) Project No. 277101999 TRR 183 (Project No. A01/A03). The Integrated Quantum Optics group acknowledges financial support through the Gottfried Wilhelm Leibniz-Preis (Grant No. SI1115/3-1) and the European Commission through the ERC project QuPoPCoRN (Grant No. 725366).

\section{APPENDIX A: EFFECTIVE FIELD THEORY}

In this Appendix, we discuss how the evaluation of the probability distributions $P_{\sigma^{\prime} \sigma}(t, q)$ at large distance scales $q \gg 1$ can be brought in the framework of the effective supersymmetric (SUSY) field theory as discussed in the main text. We start by introducing a fictitious local gauge transformation of the basis defined by the unitary operator

$$
\hat{G}_{\psi}=\sum_{q=0}^{N-1}|q, \sigma\rangle e^{i \psi_{q}}\langle q, \sigma|,
$$

with $\psi_{q}=q \phi_{0}+\psi_{0}$. Here $\phi_{0}$ and $\psi_{0}$ are some constants; the phase $\psi_{q}$ is a linear ramp and, to comply with the periodic boundary conditions, we require that $\phi_{0}=2 \pi n / N$ with $n \in$ $\mathbb{Z}$. Then, by definition, $\hat{G}_{\psi}|q, \sigma\rangle=|q, \sigma\rangle e^{i \psi_{q}}$ holds true, and this enables one to represent Eq. (4) in the equivalent form

$$
P_{\sigma^{\prime} \sigma}(t, q)=\left\langle\left|\left\langle q, \sigma^{\prime}\left|G_{\psi}^{\dagger} \hat{U}^{t} G_{\psi}\right| 0, \sigma\right\rangle\right|^{2}\right\rangle_{\theta, \varphi} .
$$


This is only nominally $\psi$ dependent and the usefulness of such artificial representation is going to be evident momentarily. As one can see, the operator $\hat{G}_{\psi}$ commutes with the (local) coin operator $\hat{R}$. On the other hand, $\hat{G}_{\psi}^{\dagger} \hat{T} \hat{G}_{\psi}=e^{i \phi_{0} \sigma_{3}} \hat{T}$ applies. With this observation, we can write the probability distribution as

$$
\begin{gathered}
P_{\sigma^{\prime} \sigma}(t, q)=\left\langle\left|\left\langle q, \sigma^{\prime}\left|\left[\hat{U}_{\phi_{0}}\right]^{t}\right| 0, \sigma\right\rangle\right|^{2}\right\rangle_{\theta, \varphi}, \\
\text { where } \hat{U}_{\phi_{0}}=e^{i \frac{\hat{\varphi}}{2} \sigma_{3}} e^{i \frac{\hat{\theta}}{2} \sigma_{1}} e^{i \phi_{0} \sigma_{3}} \hat{T} e^{\frac{\hat{\theta}}{2} \sigma_{1}} e^{i \frac{\hat{\varphi}}{2} \sigma_{3}},
\end{gathered}
$$

and average the latter over the auxiliary angle $\phi_{0}$.

For that, let us consider the (disorder-specific) SUSY action:

$$
S_{0}[\bar{\psi}, \psi]=\int d q^{\prime} \bar{\psi}_{q^{\prime}}\left(1-e^{i \frac{\omega}{2}-0} \hat{U}_{\phi_{0}}\right) \psi_{q^{\prime}}
$$

Here the fields $\bar{\psi}_{q}=\left\{\bar{\psi}_{q}^{\alpha, \sigma}\right\}$ are four-component supervectors, consisting of (anti)commuting components $\alpha=$ (f)b and carrying spin index $\sigma= \pm$. The latter denote the eigenvalue of a spin operator $\hat{s}_{i}$, which we here keep rather general, i.e., $i=1,2,3$, although our focus was on the chiral operator $i=2$ in the main text. The notation $\int d q$ above is symbolic in the sense that operator $\hat{U}_{\phi_{0}}$, in fact, maps the spinor $\psi_{q}$ onto $\psi_{q \pm 1}$. On taking into account the chiral symmetry, $\hat{G}_{-\epsilon}^{A}=\sigma_{2} G_{\epsilon}^{R} \sigma_{2}$, the probability in Eq. (14) can be then obtained via a Gaussian functional average:

$$
\begin{aligned}
& P_{\sigma^{\prime} \sigma}^{\text {chiral }}(\omega, q) \\
& \quad=|\omega|\left\langle\int \mathcal{D}(\bar{\psi}, \psi) \psi_{q}^{\mathrm{b} \sigma^{\prime}} \bar{\psi}_{0}^{\mathrm{b} \sigma}\left[\sigma_{2} \psi_{0}^{\mathrm{f}}\right]^{\sigma}\left[\bar{\psi}_{q}^{\mathrm{f}} \sigma_{2}\right]^{\sigma^{\prime}} e^{-S_{0}[\bar{\psi}, \psi]}\right\rangle_{\theta, \varphi}
\end{aligned}
$$

(here we used $\left.\left(1-e^{i \frac{\omega}{2}-0} \hat{U}_{\phi_{0}}\right) \equiv\left[G_{\frac{\omega}{2}}^{R}\right]^{-1}\right)$. To facilitate the subsequent derivation, it is advantageous to augment the action by a source term:

$$
S_{J}[\bar{\psi}, \psi]=S_{0}[\bar{\psi}, \psi]-\int d q^{\prime} \bar{\psi}_{q^{\prime}}\left(j_{q^{\prime}} \sigma_{2}\right) \psi_{q^{\prime}}
$$

Here the current $j_{q^{\prime}}=\alpha \delta_{q^{\prime} 0} \pi^{\mathrm{bf}} \otimes \pi_{i}^{\sigma}+\beta \delta_{q^{\prime} q} \pi^{\mathrm{fb}} \otimes \pi_{i}^{\sigma^{\prime}}$ involves projection matrices in spin and graded space, $\pi_{i}^{\sigma}=$ $\frac{1}{2}\left(1+\sigma \sigma_{i}\right), \pi^{\mathrm{bf}}=\left(\begin{array}{ll}0 & 1 \\ 0 & 0\end{array}\right), \pi^{\mathrm{fb}}=\left(\begin{array}{ll}0 & 0 \\ 1 & 0\end{array}\right)$, and affords a calculation of the probability distribution according to

$$
P_{\sigma^{\prime} \sigma}^{\text {chiral }}(\omega, q)=\left.|\omega|\left\langle\partial_{\alpha \beta}^{2} \mathcal{Z}_{J}\right\rangle_{\theta, \varphi}\right|_{\alpha=\beta=0},
$$

where $\mathcal{Z}_{J}$ is a partition sum of the action $S_{J}[\bar{\psi}, \psi]$. This identity can be checked by a straightforward computation which invokes the Wick's theorem for the Gaussian action $S_{0}$.

The probability in Eq. (A6) is $\phi_{0}$ independent by construction. Thus, one can average the generating function over all equivalent gauge configurations, $\left\langle\mathcal{Z}_{J}\right\rangle_{\phi_{0}}=\int_{0}^{2 \pi} \frac{d \phi_{0}}{2 \pi} \mathcal{Z}_{J}$. This integral can be done via the color flavor transformation [74] by trading the microscopic degrees of freedom $\bar{\psi}_{q}, \psi_{q}$ for bilocal matrix fields $\bar{Z}_{q q^{\prime}}, Z_{q q^{\prime}}$, representing the Goldstone, viz. diffusion modes, of the disordered single-particle system. To see its working principle, let us decompose the Floquet evolution operator as

$$
U_{\phi_{0}}=V_{1}(\theta, \varphi) T_{+} e^{i \phi_{0} \sigma_{3}} T_{-} V_{2}(\theta, \varphi),
$$

where partial coin rotations are $V_{1}(\varphi, \theta)=e^{i \frac{\varphi q}{2} \sigma_{3}} e^{i \frac{\theta_{q}}{2} \sigma_{1}}$ and $V_{2}(\varphi, \theta)=e^{i \frac{\theta_{q}}{2} \sigma_{1}} e^{i \frac{\varphi_{q}}{2} \sigma_{3}}$, while $T_{\sigma}=T \pi_{3}^{\sigma}+\pi_{3}^{-\sigma}$ are shift operators describing individual hopping of the spin-up and -down particles to the left and right, respectively. If one further introduces auxiliary spinors

$$
\begin{aligned}
& \left(\psi_{1}^{T}, \psi_{2}^{T}\right)=\bar{\psi} e^{i \frac{\omega}{2}} V_{1}(\theta, \varphi) T_{+}, \\
& \left(\psi_{2}^{\prime}, \psi_{1}^{\prime}\right)^{T}=T_{-} V_{2}(\theta, \varphi) \psi,
\end{aligned}
$$

where the two-component structure refers to the spin subspace, then the free action $S_{0}$ can be cast into the equivalent form

$$
S_{0}[\psi, \bar{\psi}]=\int d q\left(\bar{\psi}_{q} \psi_{q}-\psi_{1, q}^{T} e^{i \phi_{0}} \psi_{2, q}^{\prime}-\psi_{2, q}^{T} e^{-i \phi_{0}} \psi_{1, q}^{\prime}\right) .
$$

At the heart of the color-flavor transformation lies the identity $[74,75]$

$$
\begin{aligned}
& \int_{0}^{2 \pi} \frac{d \phi_{0}}{2 \pi} e^{\sum_{q}\left(\psi_{1, q}^{T} e^{i \phi_{0}} \psi_{2, q}^{\prime}+\psi_{2, q}^{T} e^{-i \phi_{0}} \psi_{1, q}^{\prime}\right)} \\
& \quad=\int d Z d \bar{Z} \operatorname{sdet}(1-\bar{Z} Z) e^{\sum_{q q^{\prime}}\left(\psi_{1, q}^{T} Z_{q q^{\prime}} \psi_{1, q^{\prime}}^{\prime}+\psi_{2, q}^{T} \bar{Z}_{q q^{\prime}} \psi_{2, q^{\prime}}^{\prime}\right)},
\end{aligned}
$$

and sdet refers to the graded determinant. Here $\bar{Z}=\left\{\bar{Z}^{\alpha \alpha^{\prime}}\right\}$ and $Z=\left\{Z^{\alpha \alpha^{\prime}}\right\}$ are the (graded) matrix fields mentioned above, with components $\alpha, \alpha^{\prime} \in\{\mathrm{b}, \mathrm{f}\}, \bar{Z}^{\mathrm{bb}}=-\left[Z^{\mathrm{bb}}\right]^{\dagger}$ and $\bar{Z}^{\mathrm{ff}}=\left[Z^{\mathrm{ff}}\right]^{\dagger}$ to guarantee convergence of Eq. (A12), and the additional matrix structure of $Z$ is in spin space. The anticommuting blocks $Z^{\alpha \alpha^{\prime}}$ and $\bar{Z}^{\alpha \alpha^{\prime}}$ with $\alpha \neq \alpha^{\prime}$ are independent variables. On applying this identity to the partition $\operatorname{sum}\left\langle\mathcal{Z}_{J}\right\rangle_{\phi_{0}}$ and then integrating over fields $\left(\bar{\psi}_{q}, \psi_{q}\right)$, we can reduce the former to the path integral over collective matrix fields $(\bar{Z}, Z)$ with an action

$$
\begin{aligned}
S[\bar{Z}, Z]= & -\operatorname{str} \ln (1-\bar{Z} Z) \\
& +\operatorname{str} \ln \left(1-e^{i \frac{\omega}{2}} V_{1} T_{+}\left(\begin{array}{cc}
0 & Z \\
\bar{Z} & 0
\end{array}\right) T_{-} V_{2}-j \sigma_{2}\right) .
\end{aligned}
$$

Recalling the chiral symmetry, $\sigma_{2} T_{-} V_{2} \sigma_{2}=\left(T_{+}\right)^{\dagger} V_{1}^{\dagger}$, one identifies two Goldstone modes of this action, $Z=-\bar{Z}$, whenever $\omega \rightarrow 0$ or $2 \pi$ are satisfied (this corresponds to particle/hole energies $\pm \epsilon$ being close to 0 or $\pm \pi$, respectively). Indeed, if $Z=-\bar{Z}$ are constant in space and $j=0$, then action Eq. (A13) vanishes identically. Physically, the field $Z_{q q^{\prime}}^{\alpha \alpha^{\prime}} \sim \psi_{q}^{\alpha} \bar{\psi}_{q^{\prime}}^{\alpha^{\prime}}$ describes a pairwise propagation of a retarded and an advanced single-particle amplitude at a slight $(\bmod 2 \pi)$ difference in frequency $\omega$. At long spatial scales, off-diagonal components (with $q \neq q^{\prime}$ ) relax quickly due to accumulation of random phases, and Goldstone modes assume the form $Z_{q q^{\prime}}=Z_{q} \delta_{q q^{\prime}}$. Assuming $Z_{q}$ to vary slowly in space, we further expand Eq. (A13) in small spatial gradients and frequency.

\section{Topological and second-order gradient terms}

Let us first discuss terms with spatial derivatives and set $j=\omega=0$ - this corresponds to the first Goldstone modeand we comment on the second one (with $\omega \rightarrow 2 \pi$ ) in the 
end of this Appendix. By defining $\mathcal{Z}=\left(1-i Z \sigma_{2}\right)$, one can rewrite the action Eq. (A13) as

$$
S[Z]=-\operatorname{str} \ln (\mathcal{Z})+\operatorname{str} \ln (\mathcal{Z}+\delta \mathcal{Z}),
$$

with

$$
\delta \mathcal{Z}=-V_{1} T_{+}\left[Z, T_{+}^{\dagger}\right] V_{1}^{\dagger} i \sigma_{2} .
$$

To second order,

$$
\begin{aligned}
S[Z] & \simeq \operatorname{Str}\left(\mathcal{Z}^{-1} \delta \mathcal{Z}\right)-\frac{1}{2} \operatorname{Str}\left(\mathcal{Z}^{-1} \delta \mathcal{Z} \mathcal{Z}^{-1} \delta \mathcal{Z}\right)+\ldots \\
& =S^{(1)}[Z]+S^{(2)}[Z]+\ldots,
\end{aligned}
$$

while $\langle q|\left[T_{+}\left[Z, T_{+}^{\dagger}\right]|q\rangle=\left(Z_{q}^{\prime}+\frac{1}{2} Z_{q}^{\prime \prime}\right) P_{+} \ldots\right.$ The topological and so-called Gade terms originate from the first-order terms in these series. Using that $\mathcal{Z}^{-1}=\left(1+i \sigma_{2} Z\right) /\left(1+Z^{2}\right)$ and evaluating traces in the spin subspace, one arrives at

$$
\begin{aligned}
\left\langle S_{1}^{(1)}\right\rangle_{\theta, \varphi} & =\bar{\chi}^{0} \int d q \operatorname{str}\left(g^{-1} \partial_{q} g\right)-\int d q \partial_{q} \operatorname{str} \ln (1+g) \\
& \equiv S_{\text {top }}+S_{\mathrm{r}}
\end{aligned}
$$

Here

$$
\bar{\chi}^{0}=\frac{1}{2}\left(1-\left\langle\sin \theta_{q} \cos \varphi_{q}\right\rangle_{\theta, \varphi}\right),
$$

and we introduced $g=(1+i Z) /(1-i Z)$. Geometrically, the unconstrained pair $(\bar{Z}, Z)$ defines a set of stereographic coordinates parametrizing a two-dimensional sphere in the fermionic ff sector, respectively, hyperboloid in the bosonic bb sector. This is readily verified, recalling that $\bar{Z}^{\mathrm{ff} / \mathrm{bb}}=$ $\pm\left[Z^{\mathrm{ff} / \mathrm{bb}}\right]^{*}$ and stereographic coordinates

$$
\left(x_{1}, x_{2}, x_{3}\right)=\frac{1}{1 \pm \bar{z} z}( \pm 2 \operatorname{Re} z, \mp 2 \operatorname{Im}(z), 1 \mp \bar{z} z)
$$

for the two-sphere and hyperboloid, respectively. The Goldstone-mode restriction $\bar{Z}=-Z$ defines 1D submanifolds which result from their intersection with two-dimensional planes, viz. a circle, respectively, hyperbola. The latter identifies $g \in \mathrm{Gl}(1 \mid 1)$ as a supersymmetric group manifold.

For a system with periodic boundary conditions, we can omit the second (residual) term $S_{r}$ and keep only the first (topological) one. In fact, both terms are full derivatives since $\operatorname{str}\left(g^{-1} \partial_{q} g\right)=\partial_{q} \ln \operatorname{det}(g)$. However, $S_{\text {top }}$ is nontrivial. Consider a configuration $g=\left(\begin{array}{cc}e^{x} & 0 \\ 0 & 1\end{array}\right)_{\mathrm{bf}}$, where $x$ is a compact fermion angle. Assuming periodic boundary conditions, mappings $x_{q}: S_{1} \rightarrow S_{1}$ may have windings, i.e., $x_{L}=x_{0}+2 \pi W$, where $W \in \mathbb{Z}$. Then action $S_{\text {top }}$ on such configuration becomes nonzero, $S_{\text {top }}=2 i \pi n \chi$. For the residual term, one finds

$$
S_{r}=\int_{0}^{2 \pi n} \frac{i e^{i x}}{1+e^{i x}} d x=n \oint_{|w|=1} \frac{d w}{(1+w)} .
$$

If one regularizes this integral by slightly shifting the pole $w=1$ outside the unit circle $|w|=1$, then $S_{r}$ vanishes.

The Gade term is obtained if one keeps the second-order cumulant expansion when averaging over disorder,

$$
S_{\mathrm{G}}[g]=-\frac{1}{2} c \int d q \operatorname{str}^{2}\left(g^{-1} \partial_{q} g\right),
$$

where $c=\left\langle\left\langle\chi^{2}(\theta, \varphi)\right\rangle\right\rangle=\left\langle\chi^{2}(\theta, \varphi)\right\rangle_{\theta, \varphi}-\bar{\chi}^{2}$. This term is exactly zero at criticality (where $\chi=\frac{1}{2}$ and does not fluctuate) and is known to give inessential modifications away from it [76].

Since in this paper we are interested in critical quantum walks only, we can derive the diffusive action $S_{0}[g]$ by setting $V_{1}=V_{2}=\mathbb{1}$ (this corresponds to $\theta=0, \pi$ ). The latter simplifies the variation, $\delta \mathcal{Z}=-\left(Z_{q}^{\prime}+\frac{1}{2} Z_{q}^{\prime \prime}\right) P_{+} i \sigma_{2} \ldots$, and action $S_{0}[g]$ originates from two pieces. The first piece is

$$
S^{(2)}[Z]=-\frac{1}{2} \operatorname{str}\left(\left(1+Z^{2}\right)^{-1} Z Z^{\prime}\right)^{2},
$$

while the second piece stems from the second-order gradient term $\left(\propto Z^{\prime \prime}\right)$ in $S^{(1)}[Z]$. It evaluates to

$$
S_{2}^{(1)}[Z]=\frac{1}{2} \operatorname{str}\left(\left(1+Z^{2}\right)^{-1} Z Z^{\prime \prime}\right) .
$$

By adding these two contributions and integrating by parts one finds the diffusive action of the class AIII $\sigma$ model:

$$
S_{0}[g]=-\frac{1}{2} \operatorname{str}\left(\frac{1}{1+Z^{2}} Z^{\prime}\right)^{2}=-\frac{1}{8} \int d q \operatorname{str}\left(\partial_{q} g^{-1} \partial_{q} g\right) .
$$

Let us now comment on the second Goldstone mode with $\omega \rightarrow 2 \pi$. If we change $Z \rightarrow-Z$ in the prototype action Eq. (A13), it is reduced to the one with the first Goldstone $(\omega \rightarrow 0)$ and at the same time $g \rightarrow g^{-1}$. The latter does not change $S_{0}[g]$ but transforms the topological angle, $\bar{\chi}^{0} \rightarrow \bar{\chi}^{\pi}=1-\bar{\chi}^{0}$, in the action Eq. (A17). At criticality, both Goldstone modes are described by the same action with $\bar{\chi}=1 / 2$.

To summarize, using supersymmetric techniques for disordered systems [52] and the color-flavor transformation [74,75], we arrive at the effective action, $S^{\epsilon}=S_{0}+S_{\text {top }}^{\epsilon}$, where

$$
\begin{aligned}
& S_{0}=\frac{1}{2} \int d q\left[-\mathfrak{g}_{0} \operatorname{str}\left(\partial_{q} g^{-1} \partial_{q} g\right)+i \omega \operatorname{str}\left(g+g^{-1}\right)\right], \\
& S_{\text {top }}^{\epsilon}=\bar{\chi}^{\epsilon} \int d q \operatorname{str}\left(g^{-1} \partial_{q} g\right) .
\end{aligned}
$$

Here $g$ denotes a group-valued matrix field that describes the critical fluctuations in the system, $\mathfrak{g}_{0}=1 / 4$ is the bare conductance, and

$$
\bar{\chi}^{\epsilon}=\frac{1}{2}\left(1-e^{i \epsilon}\langle\sin (\theta) \cos (\varphi)\rangle_{\theta, \varphi}\right)
$$

is the bare topological angle with $\epsilon=0, \pi$ indicating the critical states described by the effective action. Its is also worth mentioning here that the action $S^{\epsilon}$ is identical to the one describing disordered quantum wires of a symmetry class AIII [10].

\section{a. Sources}

Finally let us turn to source contributions $S_{J}$ to the action. Relevant contributions result from an expansion of the action Eq. (A13) to linear order in $j$,

$$
\begin{aligned}
S_{J} & =-\operatorname{str}\left(j \sigma_{2} \mathcal{Z}^{-1}\right) \\
& =-\alpha\left[\frac{i Z_{0}+\sigma \delta_{i 2}}{1+Z_{0} Z_{0}}\right]^{\mathrm{fb}}-\beta\left[\frac{i Z_{q}+\sigma^{\prime} \delta_{i 2}}{1+Z_{q} Z_{q}}\right]^{\mathrm{bf}}
\end{aligned}
$$


or

$$
S_{J}[g]=-\left\{\begin{array}{l}
\frac{\alpha}{4}\left[g_{0}-g_{0}^{-1}\right]^{\mathrm{fb}}+\frac{\beta}{4}\left[g_{q}-g_{q}^{-1}\right]^{\mathrm{bf}}, i=1,3 \\
\frac{\sigma \alpha}{2}\left[g_{0}^{\sigma}\right]^{\mathrm{fb}}+\frac{\sigma^{\prime} \beta}{2}\left[g_{q}^{\sigma^{\prime}}\right]^{\mathrm{bf}}, i=2,
\end{array}\right.
$$

resulting in

$$
\begin{gathered}
P_{\sigma^{\prime} \sigma}^{\text {chiral }}=\sum_{\sigma, \sigma^{\prime}= \pm} \frac{\sigma \sigma^{\prime}}{16}|\omega|\left\langle\left\langle\left[g_{0}^{\sigma}\right]^{\mathrm{fb}}\left[g_{q}^{\sigma^{\prime}}\right]^{\mathrm{bf}}\right\rangle\right\rangle, \quad i=1,3, \\
P_{\sigma^{\prime} \sigma}^{\text {chiral }}=\frac{\sigma \sigma^{\prime}}{4}|\omega|\left\langle\left\langle\left[g_{0}^{\sigma}\right]^{\mathrm{fb}}\left[g_{q}^{\sigma^{\prime}}\right]^{\mathrm{bf}}\right\rangle\right\rangle, \quad i=2,
\end{gathered}
$$

We evaluate these propagators in the next Appendix.

\section{APPENDIX B: TRANSFER MATRIX METHOD}

When evaluating the probability distributions Eqs. (A29) and (A30), the nonperturbative nature of Anderson localization requires the functional integration over the entire group manifold, which usually is a highly complicated task. We are here, however, in a better situation since powerful alternative nonperturbative methods are available for the 1D $\sigma$ model $[52,77]$. The latter is based on the interpretation of the action $S_{0}[g]$ as the action of a quantum mechanical particle with coordinate $g$ moving in the potential $V(g)=\eta \operatorname{str}\left(g+g^{-1}\right)$ where $\eta=-i \omega$. Changing then from the path-integral to the Schrödinger description, one expresses the probability distribution in a spectral decomposition with respect to the corresponding Hamilton operator

$$
\hat{H}=\Delta_{g}+V(g)
$$

where $\Delta_{g}=-J^{-1} \partial_{i} G^{i j} J \partial_{j}$ is the Beltrami-Laplace operator on the AIII manifold, with metric tensor $G^{i j}$ and Jacobian $J=$ $\sqrt{\operatorname{sdet} G}$

In what follows, we sketch the details of such a program at criticality when $\bar{\chi}=1 / 2$ and derive a propagator of the quantum Sinai diffusion. We start by parametrizing the field $g$ in terms of four coordinates $z=(x, y, \bar{\xi}, \xi)$ such that

$$
g=\mathcal{U}\left(\begin{array}{cc}
e^{x} & 0 \\
0 & e^{i y}
\end{array}\right)_{\mathrm{bf}} \mathcal{U}^{-1}, \quad \mathcal{U}=\exp \left(\begin{array}{ll}
0 & \xi \\
\xi & 0
\end{array}\right)_{\mathrm{bf}},
$$

with $x, y \in \mathbb{R}$ being commutative while $\bar{\xi}$, $\xi$ being Grassmann anticommutative fields, which results in the following metric:

$$
\begin{aligned}
d l^{2} & =-\operatorname{str}\left(d g d g^{-1}\right)=G_{i j} d z^{i} d z^{j} \\
& =d x^{2}+d y^{2}+8 \sinh ^{2}\left(\frac{x-i y}{2}\right) d \bar{\xi} d \xi
\end{aligned}
$$

on the GL(1|1) manifold. Equation (B4) above defines nonzero elements of the tensor $G_{i j}$. With the Jacobian $J(z)=$ $\frac{1}{4} \sinh ^{-2}\left(\frac{x-i y}{2}\right)$ and the vector potential $A=\bar{\chi}(i, 1,0,0)$ this metric defines the transfer matrix Hamiltonian

$$
\mathcal{H}=-J^{-1}(z)\left(\partial_{\mu}-i A_{\mu}\right) G^{\mu \nu} J(z)\left(\partial_{\nu}-i A_{\nu}\right)+V(z),
$$

where $V(x, y)=\eta(\cosh x-\cos y)$ is the potential energy due to the frequency term in the action and $\eta=-i \omega$. Then the Sutherland transformation,

$$
H=e^{\bar{\chi}(x-i y)} J^{1 / 2} \mathcal{H} J^{-1 / 2} e^{-\bar{\chi}(x-i y)},
$$

complemented by the gauge transform eliminating the vector potential, brings the Hamiltonian to a simpler form:

$$
\hat{H}=-\partial_{x}^{2}-\partial_{y}^{2}-\frac{1}{2} \sinh ^{-2}\left(\frac{x-i y}{2}\right) \partial_{\bar{\xi}} \partial_{\xi}+V(x, y) .
$$

The ground state $|0\rangle \equiv \Phi_{0}(x, y)$ of $\hat{H}$-it obeys $\hat{H}|0\rangle=0$ due to supersymmetry-depends only on bosonic angles $(x, y)$ and can be approximated by

$$
\Phi_{0}(x, y)=-\operatorname{coth}\left(\frac{x-i y}{2}\right) K_{0}\left(\sqrt{2 \eta} e^{|x| / 2}\right) / \ln \eta .
$$

If $\eta \ll 1$, then the latter correctly interpolates between the two analytically known expressions for the ground state $|0\rangle$ in the limit $x \sim 1$ and $|x| \gg 1$, respectively [75]. The excited states $|k\rangle \equiv \Phi_{k}(z)$ of $\hat{H}$ with energies $E_{k}>0$ can be labeled by a set of quantum numbers $k=(n, l, \bar{\lambda}, \lambda)$, where $n$ and $l$ are integers and $\bar{\lambda}, \lambda$ are Grassmanns. Specifically,

$$
\Phi_{k}(z)=\mathcal{R}_{k}(x, y) \times e^{\bar{\xi} \lambda+\xi \bar{\lambda}}
$$

can be split into radial and angular parts where $\mathcal{R}_{k}(x, y)$ satisfies to the radial Schrödinger equation

$$
\left(-\partial_{x}^{2}-\partial_{y}^{2}+V(x, y)-\frac{1}{2} \sinh ^{-2}\left(\frac{x-i y}{2}\right) \bar{\lambda} \lambda\right) \mathcal{R}_{k} \Phi_{k}=E_{k} \mathcal{R}_{k} .
$$

Since $\bar{\lambda} \lambda$ is the nilpotent of the Grassmann algebra, the spectrum and eigenstates of the above radial equation should have the following form: $E_{k}=\epsilon_{n, l}+\bar{\lambda} \lambda \epsilon_{n, l}^{\prime}$ and

$$
\mathcal{R}_{k}(x, y)=R_{n, l}(x, y)+\bar{\lambda} \lambda R_{n, l}^{\prime}(x, y),
$$

where $n=1,2, \ldots$, and $l \in \mathbb{Z}$ are radial quantum numbers. It turns out (see sub sub section of Appendix B below) that only the zeroth-order terms in bilinear $\bar{\lambda} \lambda$ are required to evaluate the propagator $P_{\sigma^{\prime} \sigma}^{\text {chiral }}(\eta, q)$ of the quantum Sinai diffusion. We proceed by constructing an asymptotic form of the radial wave function $R_{n, l}(x, y)$ at $\eta \ll 1$ (or $t \gg 1$ ) in the next section and then find $P_{\sigma^{\prime} \sigma}$ in sub sub section of Appendix B.

\section{Radial wave function}

We now concentrate on the spectrum $\epsilon_{n, l}$ and eigenstates $R_{n, l}(x, y)$ of the zeroth-order Hamiltonian:

$$
\hat{H}_{0}=-\partial_{x}^{2}-\partial_{y}^{2}+\eta(\cosh x-\cos y) .
$$

It will be seen in sub sub section of Appendix B that in the limit $\eta \ll 1$ which we are going to explore essential $x$ 's satisfy $\eta e^{|x|} \sim 1$ and hence $\cos y$ term in $\hat{H}_{0}$ can be neglected. We thus approximate $R_{n, l}(x, y) \approx R_{n}(x) e^{i l y}$, which leads to $\epsilon_{n, l}=$ $\epsilon_{n}+l^{2}$ together with a simple radial equation

$$
\left[-\partial_{x}^{2}+\eta \cosh x\right] R_{n}(x)=\epsilon_{n} R_{n}(x) .
$$

To solve it, we introduce momenta $k_{n}=\sqrt{\epsilon_{n}}$ and divide the $x$ axis in three intervals: (i) small angles with $|x|<1$, (ii) intermediate ones, such that $1<|x|<\ln (1 / \eta)$, and (iii) large angles, where $|x|>\ln (1 / \eta)$. In the following, it will be sufficient to consider the domain $x>0$ since the potential $\cosh x$ is symmetric. In the intervals ii and iii, one can approximate Eq. (B13) by

$$
\left[-\partial_{x}^{2}+\frac{1}{2} \eta e^{x}\right] R_{n}(x)=k_{n}^{2} R_{n}(x) .
$$


Up to a normalization factor which is found below, the solution of this equation is a modified Bessel function $R_{n}(x) \propto$ $K_{2 i k_{n}}\left(\sqrt{2 \eta} e^{x / 2}\right)$. Taking a limit of $K_{v}(z)$ at small argument, the wave function $R_{n}(x)$ in interval ii is reduced to the plane wave

$$
\begin{aligned}
R_{n}(x) & \propto A\left(k_{n}\right) e^{i k_{n} x}+A^{*}\left(k_{n}\right) e^{-i k_{n} x}, \\
A(k) & =\Gamma(-2 i k)(\eta / 2)^{i k} .
\end{aligned}
$$

As for interval $\mathrm{i}$, one can neglect the $\eta$-dependent potential whatsoever and therefore by continuity the plane wave Eq. (B15) is also a solution in interval $\mathrm{i}$. We can thus introduce a scattering matrix and a phase shift from the right potential barrier, $S(k)=A(-k) / A(k)=e^{-i \phi(k)}$, which finally gives us a quantization condition $\phi\left(k_{n}\right)=\pi n$. Here $n=0,1,2, \ldots$ with even or odd $n$ corresponding to even or odd wave functions $R_{n}(x)$, respectively, i.e., $R_{n}(-x)=(-1)^{n} R_{n}(x)$. For small momenta, $k_{n} \ll 1$, we get with $\log$ accuracy $\eta^{2 i k} \simeq e^{i \pi(n+1)}$, which leads to the spectrum

$$
\epsilon_{n, l}=\frac{\pi^{2}}{4 \ln ^{2} \eta}(n+1)^{2}+l^{2}, \quad n=0,1,2, \ldots, l \in \mathbb{Z} .
$$

We now proceed to find a normalization factor for the radial wave function. For that, let's note that the main contribution to its norm $\int_{-\infty}^{+\infty} R_{n}^{2}(x) d x=1$ comes from the intervals $\mathrm{i}$ and ii. The wave function in these regions is a plane wave:

$$
R_{n}(x) \propto\left|A\left(k_{n}\right)\right| \cos \left(k_{n} x+\frac{1}{2} \pi n\right) .
$$

It can be matched to the one found within the semiclassical approximation,

$$
R_{n}(x)=\left(C_{n} / \sqrt{k_{n}}\right) \cos \left(k_{n} x+\frac{1}{2} \pi n\right),
$$

where the normalization constant is fixed by $C_{n}^{2}=$ $\left(2 k_{n} / \pi\right)\left(\partial k_{n} / \partial n\right)$. The comparison of these two representations leads to the following normalized radial wave function:

$$
\begin{aligned}
R_{n, l}(x, y) & =e^{i l y}\left(\frac{2}{\pi} \frac{\partial k_{n}}{\partial n}\right)^{1 / 2}\left|A\left(k_{n}\right)\right|^{-1} K_{2 i k_{n}}\left(\sqrt{2 \eta} e^{x / 2}\right), \\
|A(k)|^{-1} & =\left(\frac{2 k \sinh 2 \pi k}{\pi}\right)^{1 / 2}, \quad x>0 .
\end{aligned}
$$

We use this intermediate result in the next subsection to evaluate the series expansion of the propagator $P_{\sigma^{\prime} \sigma}^{\text {chiral }}$.

\section{Propagator of Sinai diffusion}

Employing a spectral decomposition, the propagator Eq. (7) can be written as a sum over excited eigenstates $|k\rangle$,

$$
P_{\sigma^{\prime} \sigma}^{\text {chiral }}(\eta, q)=\eta \sum_{n, l} \int d \lambda d \bar{\lambda} \Gamma_{k}^{\sigma^{\prime}} \bar{\Gamma}_{k}^{\sigma} e^{-2 E_{k}|q|},
$$

where

$$
\begin{aligned}
\Gamma_{k}^{\sigma^{\prime}} & =\left\langle 0\left|\left[g^{\sigma^{\prime}}\right]^{\mathrm{bf}}\right| k\right\rangle \\
& =\frac{\sigma^{\prime}}{4 \pi} \int_{-\infty}^{+\infty} d x \int_{0}^{2 \pi} d y \int d \bar{\xi} d \xi \Phi_{0}(x, y)\left[g^{\sigma^{\prime}}\right]^{\mathrm{bf}} \Phi_{k}(z)
\end{aligned}
$$

is a matrix element of the field $\left[g^{\sigma^{\prime}}\right]^{\mathrm{bf}}=\left(e^{i \sigma^{\prime} y}-e^{\sigma^{\prime} x}\right) \xi$ between the ground and excited states and a similar expression is valid for a conjugated matrix element $\bar{\Gamma}_{k}$ of the field $\left[g^{\sigma}\right]^{\mathrm{fb}}=$ $\left(\left[g^{\sigma}\right]^{\mathrm{bf}}\right)^{*}$. Using the explicit form of the excited state,

$$
\Phi_{k}(z)=\mathcal{R}_{k}(x, y) \times e^{\bar{\xi} \lambda+\xi \bar{\lambda}}
$$

one can first perform the integral over Grassmanns $(\bar{\xi}, \xi)$ in Eq. (B21) and verify that the nilpotent part $\sim R_{n, l}^{\prime}(x, y)$ of the radial wave function does not contribute to the matrix elements. The latter are then simplified to $\Gamma_{k}^{\sigma^{\prime}}=-\lambda \Gamma_{n, l}^{\sigma^{\prime}}$ and $\bar{\Gamma}_{k}^{\sigma}=\bar{\lambda} \Gamma_{n, l}^{\sigma}$ with

$$
\Gamma_{n, l}^{\sigma}=\frac{\sigma}{4 \pi} \int_{-\infty}^{+\infty} d x \int_{0}^{2 \pi} d y \Phi_{0}(x, y)\left(e^{\sigma x}-e^{i \sigma y}\right) R_{n, l}(x, y) .
$$

Separating here $y$-dependent parts of the wave functions, the integration over the compact angle $y$ yields

$$
\frac{\sigma}{2 \pi} \int_{-\pi}^{\pi} d y \operatorname{coth}\left(\frac{x-i y}{2}\right)\left(e^{\sigma x}-e^{i \sigma y}\right) e^{i l y}=e^{\sigma x} \delta_{l}+\delta_{l+\sigma} .
$$

Here the $l= \pm 1$ terms induce a gap in the spectrum $\left(\epsilon_{n, \pm}=\right.$ $\left.\epsilon_{n}+1\right)$, thus we keep the $l=0$ contribution only; the latter reads

$$
\Gamma_{n, 0}^{\sigma}=-\frac{1}{2} \int e^{\sigma x} K_{0}\left(\sqrt{2 \eta} e^{|x| / 2}\right) R_{n, 0}(x) d x / \ln \eta .
$$

It is worth mentioning that $R_{n, 0}(x)$ is either even or odd depending on a parity of $n$, thus $\Gamma_{n, 0}^{+}=(-1)^{n} \Gamma_{n, 0}^{-}$. On changing the integration variable to $z=\sqrt{2 \eta} e^{x / 2}$, the remaining integral for $\Gamma_{n, 0}^{\sigma}$ is reduced to a table one:

$$
\int_{0}^{+\infty} z K_{0}(z) K_{2 i k}(z) d z=\left(k^{2} \pi^{2} / 2\right) \sinh ^{-2}(\pi k) .
$$

Finally, taking into account proper normalization factors given in Eq. (B19), one finds the following matrix elements:

$$
\begin{aligned}
M_{n}^{\sigma^{\prime} \sigma} & =\Gamma_{n, 0}^{\sigma^{\prime}} \Gamma_{n, 0}^{\sigma} \\
& =\left(\sigma^{\prime} \sigma\right)^{n} \frac{\pi^{2}}{2} \frac{k_{n}^{2}}{\eta^{2} \ln ^{2} \eta}\left(\frac{\partial k_{n}}{\partial n}\right) \times \frac{k_{n}^{3} \cosh \left(\pi k_{n}\right)}{\sinh ^{3}\left(\pi k_{n}\right)} \\
& \stackrel{k_{n} \ll 1}{\longrightarrow} \frac{\left(\sigma^{\prime} \sigma\right)^{n}(n+1)^{2}}{\eta^{2} \ln ^{5}(1 / \eta)} .
\end{aligned}
$$

From here, the propagator of Sinai diffusion is constructed as

$$
\begin{aligned}
P_{\sigma^{\prime} \sigma}^{\text {chiral }}(\eta, q) & =\eta \sum_{n, l=0} \int d \lambda d \bar{\lambda} \Gamma_{k}^{\sigma^{\prime}} \bar{\Gamma}_{k}^{\sigma} e^{-2|q| E_{k}} \\
& =\eta \sum_{n=0}^{+\infty} M_{n}^{\sigma^{\prime} \sigma} e^{-2|q| \epsilon_{n, 0}} .
\end{aligned}
$$

When evaluating the above integral over Grassmann variables, one may notice that the nilpotent correction to the spectrum, $\bar{\lambda} \lambda \epsilon_{n, l}^{\prime}$, does not contribute to the net result. At large distances, $q \gg 1$, essential momenta are small, $k_{n} \ll 1$, and the Laplace transform of Eq. (B28) from $\eta$ to the time domain yields the result Eq. (18) in the main text.

As a final remark, let us evaluate the integrated probability

$$
P^{\text {chiral }}(\eta)=\sum_{\sigma^{\prime} \sigma} \int d q P_{\sigma^{\prime} \sigma}^{\text {chiral }}(\eta, q)=4 \eta \sum_{k=0}^{+\infty} \frac{M_{2 k}^{++}}{\epsilon_{2 k, 0}} .
$$



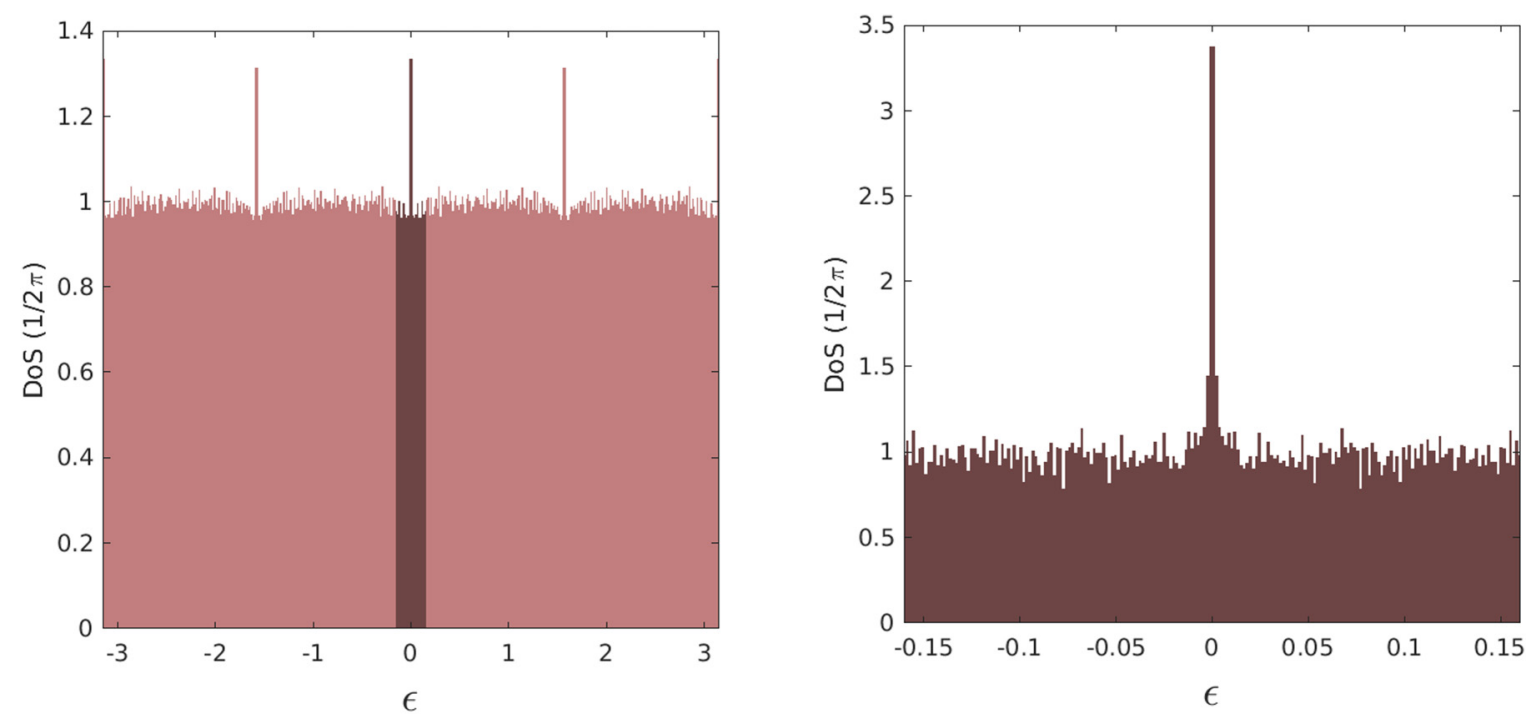

FIG. 8. Density of states (DoS) of the quantum walk model with averaged angles $(\bar{\theta}, \bar{\varphi})=(0,0)$ and disorder strengths $\gamma_{\theta}=\gamma_{\varphi}=\pi / 4$ for the quasi-energy domain $\epsilon \in[-\pi, \pi]$ (left panel) and $\epsilon \in[-0.16,0.16]$ (right panel) using different energy resolutions. In the calculations, we used averages over $10^{3}$ disorder configurations. Peaks of the DoS at chiral symmetric energy $\epsilon=0, \pm \pi / 2, \pi$ are clearly visible.

This series is convergent, owing to the exponential decay of $M_{n}^{\sigma^{\prime} \sigma}$ at large momenta $k_{n}>1$. In the limit $\eta \ll 1$, one may substitute the sum by an integral to obtain

$$
P^{\text {chiral }}(\eta)=\frac{\pi^{2}}{\eta \ln ^{2} \eta} \int_{0}^{+\infty} d k \frac{k^{3} \cosh \pi k}{\sinh ^{3} \pi k}=\frac{1}{4 \eta \ln ^{2} \eta} .
$$

Hence, the overall contribution of the critical states to the walker's probability decreases in time as $P^{\text {chiral }}(t)=$ $1 /\left(4 \ln ^{2} t\right)$.

\section{APPENDIX C: DENSITY OF STATES}

In the main text, we focused on the walker's critical dynamics at the topological Anderson localization transition. As discussed there, the critical dynamics describes quasienergy states centered around the chiral symmetric energies $\epsilon=$ $0, \pm \pi / 2, \pi$. To substantiate this statement, we provide here the numerical results for the density of states (DoS) of the quantum walker with periodic boundary conditions. Fig. 8 (left) shows the disorder averaged DoS in the entire quasienergy domain for a system of $N_{x}=400$ sites. As expected, sharp peaks are visible at the chiral symmetric energies $\epsilon=$ $0, \pm \pi / 2, \pi$. Figure 8 (right) shows a magnified view of the region colored in red of Fig. 8. With a smaller energy scale for the histogram, we can see further structures of the DoS, which is known to diverge as $\sim 1 /\left(\epsilon \ln ^{3} \epsilon\right)$ [13].

For our discussion, it is important to notice that the number of eigenstates within these energy domains is not the dominant contribution to the total DoS. This indicates that a walker initially localized on a single site is not the optimal choice for a protocol aiming to test the walker's critical dynamics, as it involves quasienergy states from the entire energy band approximately with equal weight. That is why in the main text we propose to use the plane wave with momentum $p_{0}=$ $0, \pi / 2$ as an initial state, which can be used to select only states within quasienergy regions centered around the chiral symmetric energies.

\section{APPENDIX D: TIME-STAGGERED SPIN POLARIZATION}

In this Appendix, we discuss the time-staggered spin polarization, observable in a quantum critical walk at a topological Anderson localization transition. As stated in the main text, the time-staggered spin polarization involves critical states $\epsilon \simeq \pm \pi / 2$, related to the chiral sublattice symmetry $\hat{\mathcal{C}}_{\mathrm{sl}} \equiv \sigma_{2} \otimes \hat{S}$, where $\hat{S} \equiv \sum_{q}|q\rangle(-1)^{q}\langle q|$ the sublattice operator. Before discussing the relation between $\hat{\mathcal{C}}_{\mathrm{sl}}$ and a time-staggered signal, it is instructive to reformulate our discussion in the main text on the chiral symmetry $\hat{\mathcal{C}}_{0} \equiv \hat{s}_{2}$, and related spin polarization $\Delta P$, in a more formal way which readily allows for an extension to the chiral sublattice symmetry of interest.

\section{Chiral symmetry}

In the main text, we introduced the probability distribution,

$$
P_{\sigma^{\prime} \sigma}(t, q)=\left\langle\left|\left\langle q, \sigma^{\prime}\left|\hat{U}^{t}\right| 0, \sigma\right\rangle\right|^{2}\right\rangle_{\theta, \varphi},
$$

for a walker initially prepared in eigenstate $|\sigma\rangle=|\leftarrow\rangle,|\rightarrow\rangle$ of the chiral operator $\hat{\mathcal{C}}_{0}$ to be found after $t$ time steps at a distance $q$ in eigenstate $\left|\sigma^{\prime}\right\rangle$. More formally, we can separate the walker's Hilbert space into the direct sum of subspaces characterized by the quantum numbers $s= \pm$ of the chiral operator $\hat{\mathcal{C}_{0}}, \mathcal{H}=\mathcal{H}_{+}^{0} \oplus \mathcal{H}_{-}^{0}$, and spanned by

$$
\begin{aligned}
& \mathcal{H}_{+}^{0}=\operatorname{span}\{|q, \leftarrow\rangle\}, \\
& \mathcal{H}_{-}^{0}=\operatorname{span}\{|q, \rightarrow\rangle\} .
\end{aligned}
$$

The statement on the positive spin polarization discussed in the main text can then be restated as follows: for critical states related to the chiral symmetry $\hat{\mathcal{C}}_{0}$ the probability distributions $P_{s^{\prime} s}: \mathcal{H}_{s} \stackrel{\hat{U}^{t}}{\longrightarrow} \mathcal{H}_{s^{\prime}}$ for initial and final states belonging to the same and different subspaces, $s^{\prime}=s$, respectively, $s^{\prime}=-s$, 
differ and their difference is strictly positive:

$$
P_{s s}(t, q)-P_{-s s}(t, q)>0 .
$$

Formulated in terms of quantum numbers of the chiral operator, the statement on the positivity Eq. (D4) holds for critical states related to the chiral symmetry, independently of its specific form. For the specific chiral symmetry $\hat{\mathcal{C}}_{0}=\hat{s}_{2}$ quantum numbers are simply spin orientations, and the positive difference is indeed equivalent to the positive spin polarization distribution, $P_{\rightarrow \rightarrow}^{\text {chiral }}(t, q)-P_{\leftarrow}^{\text {chiral }}(t, q) \equiv \Delta P(t, q)>$ 0 , discussed in the main text. Statement Eq. (D4) can now be applied to the chiral sublattice symmetry $\hat{\mathcal{C}}_{\mathrm{sl}}$, where it shows more interesting consequences.

Chiral sublattice symmetry:-Separating the walker's Hilbert space into the direct sum of subspaces, $\mathcal{H}=\mathcal{H}_{+}^{\text {sl }} \oplus$ $\mathcal{H}_{-}^{\text {sl }}$, characterized by the quantum numbers $s= \pm$ of the chiral sublattice symmetry $\hat{\mathcal{C}}_{\mathrm{sl}} \equiv \sigma_{2} \otimes \hat{S}$, we notice that quantum numbers differ from the spin orientations, and subspaces are now spanned by

$$
\begin{aligned}
& \mathcal{H}_{+}^{\mathrm{sl}}=\operatorname{span}\{|2 q, \leftarrow\rangle,|2 q-1, \rightarrow\rangle\}, \\
& \mathcal{H}_{-}^{\mathrm{sl}}=\operatorname{span}\{|2 q, \rightarrow\rangle,|2 q-1, \leftarrow\rangle\} .
\end{aligned}
$$

Positivity Eq. (D4) holds for critical states related to the chiral operator independently of its specific form and we next have to relate this statement to the spin polarization. The relation is more involved for $\hat{\mathcal{C}}_{\mathrm{sl}}$ than for $\hat{\mathcal{C}}_{0}$, since the spin structure of eigenstates of the former alternates between even and odd sites. More specifically, this implies that the spin structure of $P_{s^{\prime} s}$ depends on the (parity of the) propagated distance $q$, i.e.,

$$
\begin{aligned}
P_{s s}(t, q) & =\left\{\begin{array}{lr}
P_{\sigma \sigma}(t, q), & q \text { even } \\
P_{-\sigma \sigma}(t, q), & q \text { odd },
\end{array}\right. \\
P_{-s s}(t, q) & =\left\{\begin{array}{lr}
P_{-\sigma \sigma}(t, q), & q \text { even } \\
P_{\sigma \sigma}(t, q), & q \text { odd },
\end{array}\right.
\end{aligned}
$$

where $s, s^{\prime}$ are the eigenvalues of $\hat{\mathcal{C}}_{\mathrm{sl}}$ and $\sigma, \sigma^{\prime}$ those of $\sigma_{2}$.

To structure then above probabilities Eqs. (D7) according to the parity of propagated time steps $t$, we notice that the single time-step evolution $\hat{U}$ propagates states by exactly one lattice site. Starting, e.g., from the even site $q=0$ and propagating for an even number of time steps $t$ one, therefore, ends again on an even site. For an odd number of time steps $t$, on the other hand, one ends on an odd site. That is,

$$
\begin{gathered}
\left.\operatorname{span}\{|2 q, \sigma\rangle\rangle\} \stackrel{\hat{U}^{2 t}}{\longrightarrow} \operatorname{span}\{|2 q, \sigma\rangle\rangle\right\}, \\
\left.\operatorname{span}\{|2 q, \sigma\rangle\rangle\} \stackrel{\hat{U}^{2 t+1}}{\longrightarrow} \operatorname{span}\{|2 q+1, \sigma\rangle\rangle\right\},
\end{gathered}
$$

and we can relate probabilities Eqs. (D7) to the parity of propagated steps $t$ as follows. For even numbers of time steps probabilities $P_{s^{\prime} s}: \mathcal{H}_{s}^{\mathrm{sl}} \stackrel{\hat{U}^{2 t}}{\longrightarrow} \mathcal{H}_{s^{\prime}}^{\mathrm{sl}}$, conserving (changing) the quantum number of the chiral sublattice operator coincides with probabilities preserving (changing) spin orientation, $P_{s^{\prime} s}=P_{\sigma^{\prime} \sigma}$. The difference Eq. (D4) is again the spin polarization, $P_{s s}(t, q)-P_{-s s}(t, q)=P_{\rightarrow \rightarrow}^{\text {chiral }}(t, q)-P_{\leftarrow \text { chiral }}^{\longrightarrow}(t, q)$. For odd numbers of time steps, on the other hand, probabilities $P_{s^{\prime} s}: \mathcal{H}_{s}^{\mathrm{sl}} \stackrel{\hat{U}^{2 t+1}}{\longrightarrow} \mathcal{H}_{s^{\prime}}^{\mathrm{sl}}$ conserving (changing) the quantum

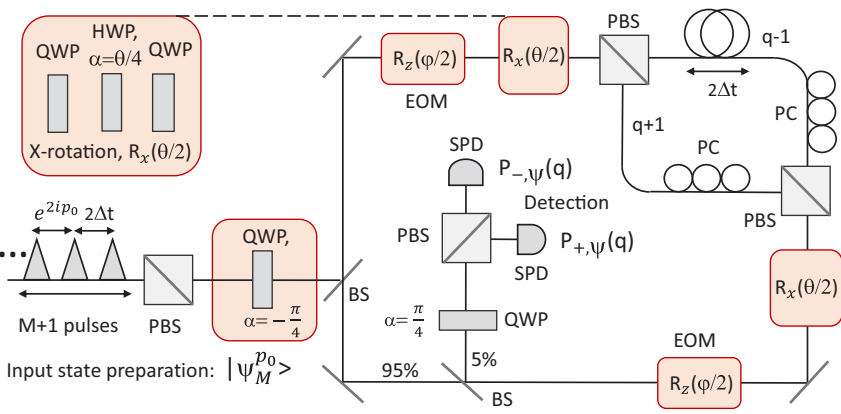

FIG. 9. A prototype of a linear optical network to realize a quantum walk discussed in details in the main text (along the lines of Refs. [21,33]). A phase modulated laser source (not shown) generates a train of pulses with a fixed time interval, $\Delta t$, and relative phase difference, $2 p_{0}$, between adjacent pulses. HWP: Half-wave plate rotated by angle $\alpha$. QWP: quarter wave plate; PBS: polarizing beam splitter; BS: beam sampler; EOM: fast switching electro-optic modulator; SPD: single-photon detector; PC: polarization controller. Fibers of different lengths ensure a time delay $2 \Delta t$ between $|H\rangle$ and $|V\rangle$ states thereby realizing shift operator $T$.

number of the chiral sublattice operator correspond to probabilities changing (preserving) spin orientation. In this case, $P_{s s}(t, q)-P_{-s s}(t, q)=P_{\leftarrow}^{\text {chiral }}(t, q)-P_{\rightarrow \rightarrow}^{\text {chiral }}(t, q)$ is the negative spin polarization distribution. Summarizing, we find that for critical states $\epsilon \simeq \pm \pi / 2$ of the chiral sublattice symmetry $\hat{\mathcal{C}}_{\text {sl }}$ positivity Eq. (D4) translates into a time-staggered spin polarization distribution,

$$
P_{\rightarrow \rightarrow}^{\text {chiral }}(t, q)-P_{\leftarrow}^{\text {chiral }}(t, q)=(-1)^{t}|\Delta P(t, q)|,
$$

as stated in the main text.

\section{APPENDIX E: DETAILS OF EXPERIMENTAL PROPOSAL}

Here we discuss few technical details related to the timemultiplexing experimental proposal mentioned in the main text, see Fig. 9. One envisions a train of equidistant pulses with controlled phase relation to be produced by a coherent laser source. The HWP and QWP plates are used for the initialization of input state in the form Eq. (25), implementation of the rotation $R_{x}(\theta)$ as well as in the detection. With a fast axis aligned horizontally, the plates in the basis of linearly polarized states, $\{|H\rangle,|V\rangle\}$, are characterized by the diagonal Jones matrices $M_{1 / 2}=\operatorname{diag}(1,-1)$ and $M_{1 / 4}=\operatorname{diag}(1, i)$. Then, for instance, the Jones matrix of the HWP rotated at $\alpha$ degrees becomes

$$
M_{1 / 2}(\alpha)=\left(\begin{array}{cc}
\cos 2 \alpha & \sin 2 \alpha \\
\sin 2 \alpha & -\cos 2 \alpha
\end{array}\right),
$$

and, on the other hand, $M_{1 / 4}(-\pi / 4) \sim R_{x}(\pi / 4)$, where the last equality holds up to inessential phase factor.

Consider now left- and right-circularly polarized states, $|L / R\rangle=\frac{1}{\sqrt{2}}(|H\rangle \pm i|V\rangle)$, which are eigenstates of the operator $\hat{\sigma}_{2}$ and thus can be identified with spin states $|\rightarrow\rangle$ and $|\leftarrow\rangle$ discussed in the main text. Assuming that a light from a laser source is linearly polarized along $|H\rangle$, one checks that $M_{1 / 4}(-\pi / 4)|H\rangle=|L\rangle$, which generates incoming state $\left|\psi_{M}^{p_{0}}\right\rangle$, cf. Eq. (25) in Sec. IV A. The same is true for the 
detection. Owing to polarizing beam splitters, two singlephoton detectors detect linearly polarized states. Because of identity $\langle R(L)|=\langle H(V)| M_{1 / 4}(\pi / 4)$, the latter are transformed into circularly polarized ones and thereby the measurement of spin-dependent probabilities $P_{\sigma \psi}(t, q)$ defined by Eq. (27) in Sec. IV A can be achieved. Finally, the identity

$$
R_{x}(\theta / 2)=M_{1 / 4} \times M_{1 / 2}(\theta / 4) \times M_{1 / 4}
$$

is a key to implement a (half) rotation along the $x$ axis using three plates as shown in Fig. 9.

Few remarks are now in order with regard to possible time and spatial scales of the experiment. Following Refs. [42,67], we assume that a laser emits a train of pulses with interval $\Delta t \sim 106 \mathrm{~ns}$ at the telecom wavelength $\lambda \sim 1550 \mathrm{~nm}$. This timing is chosen such that it is compatible with typical switching speeds of the electro-optic modulators and the deadtimes of the detectors. The intensity of such pulses in initial state should be attenuated to the single photon level, $\langle n\rangle_{\text {in }} \sim 1$, to eliminate many photon contributions in the click detectors. The interval $\Delta t$ requires a fibre length mismatch $\Delta L \sim 20 \mathrm{~m}$ between the long and short path to implement shift operator $T$ of the quantum walk. An initial wave packet of pulses with a total time span $M \Delta t$ spreads after $N$ walk's steps (each corresponding to a single run along the interferometer loop) to $(M+N) \Delta t$. Thus, for $M=10^{2}$ and $N=20$, the length of a loop should exceed $L \sim 2.5 \mathrm{~km}$, easily realizable with optical fibers in the telecom regime. Assuming that losses in the optical setup stem mainly from the coupling mismatch between in and outcoupling of the fiber and, in sum, are $20 \%$ per run, we obtain the occupation number of the order of $\langle n\rangle_{\mathrm{f}} \sim 5 \times 10^{-5}$ after $N=20$ steps. With a repetition rate of $1 \mathrm{kHz}$, this leads to 0.05 clicks per second per time bin, which should be easily detectable by superconducting singlephoton nanowire detectors within realistic measurement times. The required step numbers of 20 were already exceeded for a localized input state in Ref. [67] in which 36 steps were demonstrated. Also, the ensemble averages over 5000 realizations are in the reach of the experiment, as, e.g., in Ref. [42] 2400 disorder realizations were already measured. In summary, we strongly believe that the proposed experimental realization of the topological Anderson localization transition is feasible with current technologies and is thus in the reach of near-future measurements.
[1] K. v. Klitzing, G. Dorda, and M. Pepper, New Method for High-Accuracy Determination of the Fine-Structure Constant Based on Quantized Hall Resistance, Phys. Rev. Lett. 45, 494 (1980).

[2] D. E. Khmelnitskii, Quantization of Hall conductivity, JETP Lett. 38, 552 (1983).

[3] A. D. Mirlin, F. Evers, I. V. Gornyi, and P. M. Ostrovsky, Anderson transitions: Criticality, symmetries and topologies, Int. J. Mod. Phys. B 24, 1577 (2010).

[4] H. Obuse and N. Kawakami, Topological phases and delocalization of quantum walks in random environments, Phys. Rev. B 84, 195139 (2011).

[5] T. Rakovszky and J. K. Asboth, Localization, delocalization, and topological phase transitions in the one-dimensional splitstep quantum walk, Phys. Rev. A 92, 052311 (2015).

[6] F. Evers and A. D. Mirlin, Anderson transitions, Rev. Mod. Phys. 80, 1355 (2008).

[7] T. Ohtsuki and T. Kawarabayashi, Anomalous diffusion at the Anderson transitions, J. Phys. Soc. Jpn. 66, 314 (1997).

[8] A. Pruisken, On localization in the theory of the quantized Hall effect: A two-dimensional realization of the $\theta$-vacuum, Nucl. Phys. B 235, 277 (1984).

[9] L. Fu and C. L. Kane, Topology, Delocalization via Average Symmetry and the Symplectic Anderson Transition, Phys. Rev. Lett. 109, 246605 (2012).

[10] A. Altland, D. Bagrets, and A. Kamenev, Topology versus Anderson localization: Nonperturbative solutions in one dimension, Phys. Rev. B 91, 085429 (2015).

[11] J. Chabé, G. Lemarié, B. Grémaud, D. Delande, P. Szriftgiser, and J. C. Garreau, Experimental Observation of the Anderson Metal-Insulator Transition with Atomic Matter Waves, Phys. Rev. Lett. 101, 255702 (2008).

[12] See Ref. [78] for the recent realization of a 1D wire with chiral symmetry, where evidence for the topological Anderson insulator phase was given.
[13] L. Balents and M. P. A. Fisher, Delocalization transition via supersymmetry in one dimension, Phys. Rev. B 56, 12970 (1997).

[14] D. Bagrets, A. Altland, and A. Kamenev, Sinai Diffusion at Quasi-1D Topological Phase Transitions, Phys. Rev. Lett. 117, 196801 (2016).

[15] Y. Aharonov, L. Davidovich, and N. Zagury, Quantum random walks, Phys. Rev. A 48, 1687 (1993).

[16] D. Bouwmeester, I. Marzoli, G. P. Karman, W. Schleich, and J. P. Woerdman, Optical Galton Board, Phys. Rev. A 61, 013410 (1999).

[17] H. B. Perets, Y. Lahini, F. Pozzi, M. Sorel, R. Morandotti, and Y. Silberberg, Realization of Quantum Walks with Negligible Decoherence in Waveguide Lattices, Phys. Rev. Lett. 100, 170506 (2008).

[18] A. Peruzzo, M. Lobino, J. C. F. Matthews, N. Matsuda, A. Politi, K. Poulios, X.-Q. Zhou, Y. Lahini, N. Ismail, K. Wörhoff, Y. Bromberg, Y. Silberberg, M. G. Thompson, and J. L. OBrien, Quantum walks of correlated photons, Science 329, 1500 (2010).

[19] M. A. Broome, A. Fedrizzi, B. P. Lanyon, I. Kassal, A. AspuruGuzik, and A. G. White, Discrete Single-Photon Quantum Walks with Tunable Decoherence, Phys. Rev. Lett. 104, 153602 (2010).

[20] A. Schreiber, K. N. Cassemiro, V. Potoček, A. Gábris, P. J. Mosley, E. Andersson, I. Jex, and C. Silberhorn, Photons Walking the Line: A Quantum Walk with Adjustable Coin Operations, Phys. Rev. Lett. 104, 050502 (2010).

[21] A. Schreiber, A. Gábris, P. P. Rohde, K. Laiho, M. Štefaňák, V. Potoček, C. Hamilton, I. Jex, and C. Silberhorn, A 2D quantum walk simulation of two-particle dynamics, Science 336, 55 (2012).

[22] L. Sansoni, F. Sciarrino, G. Vallone, P. Mataloni, A. Crespi, R. Ramponi, and R. Osellame, Two-Particle Bosonic-Fermionic Quantum Walk via Integrated Photonics, Phys. Rev. Lett. 108, 010502 (2012). 
[23] A. Crespi, R. Osellame, R. Ramponi, V. Giovannetti, R. Fazio, L. Sansoni, F. De Nicola, F. Sciarrino, and P. Mataloni, Anderson localization of entangled photons in an integrated quantum walk, Nat. Photonics 7, 322 (2013).

[24] F. Cardano, F. Massa, H. Qassim, E. Karimi, S. Slussarenko, D. Paparo, C. de Lisio, F. Sciarrino, E. Santamato, R. W. Boyd, and L. Marrucci, Quantum walks and wavepacket dynamics on a lattice with twisted photons, Sci. Adv. 1, e1500087 (2015).

[25] P. Xue, R. Zhang, Z. Bian, X. Zhan, H. Qin, and B. C. Sanders, Localized state in a two-dimensional quantum walk on a disordered lattice, Phys. Rev. A 92, 042316 (2015).

[26] H. Schmitz, R. Matjeschk, C. Schneider, J. Glueckert, M. Enderlein, T. Huber, and T. Schaetz, Quantum Walk of a Trapped Ion in Phase Space, Phys. Rev. Lett. 103, 090504 (2009).

[27] F. Zähringer, G. Kirchmair, R. Gerritsma, E. Solano, R. Blatt, and C. F. Roos, Realization of a Quantum Walk with One and Two Trapped Ions, Phys. Rev. Lett. 104, 100503 (2010).

[28] M. Genske, W. Alt, A. Steffen, A. H. Werner, R. F. Werner, D. Meschede, and A. Alberti, Electric Quantum Walks with Individual Atoms, Phys. Rev. Lett. 110, 190601 (2013).

[29] M. Karski, L. Förster, J.-M. Choi, A. Steffen, W. Alt, D. Meschede, and A. Widera, Quantum walk in position space with single optically trapped atoms, Science 325, 174 (2009).

[30] P. M. Preiss, R. Ma, M. E. Tai, A. Lukin, M. Rispoli, P. Zupancic, Y. Lahini, R. Islam, and M. Greiner, Strongly correlated quantum walks in optical lattices, Science 347, 1229 (2015).

[31] J. Du, H. Li, X. Xu, M. Shi, J. Wu, X. Zhou, and R. Han, Experimental implementation of the quantum random-walk algorithm, Phys. Rev. A 67, 042316 (2003).

[32] J. Wang and K. Manouchehri, Physical Implementation of Quantum Walks (Springer, Berlin, Heidelberg, 2013).

[33] A. Schreiber, K. N. Cassemiro, V. Potoček, A. Gábris, I. Jex, and C. Silberhorn, Decoherence and Disorder in Quantum Walks: From Ballistic Spread to Localization, Phys. Rev. Lett. 106, 180403 (2011).

[34] T. Kitagawa, M. A. Broome, A. Fedrizzi, M. S. Rudner, E. Berg, I. Kassal, A. Aspuru-Guzik, E. Demler, and A. G. White, Observation of topologically protected bound states in photonic quantum walks, Nat. Commun. 3, 882 (2012).

[35] M. C. Rechtsman, J. M. Zeuner, Y. Plotnik, Y. Lumer, D. Podolsky, F. Dreisow, S. Nolte, M. Segev, and A. Szameit, Photonic Floquet topological insulators, Nature (London) 496, 196 (2013)

[36] J. M. Zeuner, M. C. Rechtsman, Y. Plotnik, Y. Lumer, S. Nolte, M. S. Rudner, M. Segev, and A. Szameit, Observation of a Topological Transition in the Bulk of a Non-Hermitian System, Phys. Rev. Lett. 115, 040402 (2015).

[37] F. Cardano, M. Maffei, F. Massa, B. Piccirillo, C. de Lisio, G. De Filippis, V. Cataudella, E. Santamato, and L. Marrucci, Statistical moments of quantum-walk dynamics reveal topological quantum transitions, Nat. Commun. 7, 11439 (2016).

[38] L. Xiao, X. Zhan, Z. Bian, K. Wang, X. Zhang, X. Wang, J. Li, K. Mochizuki, D. Kim, and N. Kawakami et al., Observation of topological edge states in parity-time-symmetric quantum walks, Nat. Phys. 13, 1117 (2017).

[39] F. Cardano, A. D’Errico, A. Dauphin, M. Maffei, B. Piccirillo, C. de Lisio, G. De Filippis, V. Cataudella, E. Santamato, and L. Marrucci et al., Detection of Zak phases and topological invariants in a chiral quantum walk of twisted photons, Nat. Commun. 8, 15516 (2017).

[40] S. Barkhofen, T. Nitsche, F. Elster, L. Lorz, A. Gábris, I. Jex, and C. Silberhorn, Measuring topological invariants in disordered discrete-time quantum walks, Phys. Rev. A 96, 033846 (2017).

[41] X. Wang, L. Xiao, X. Qiu, K. Wang, W. Yi, and P. Xue, Detecting topological invariants and revealing topological phase transitions in discrete-time photonic quantum walks, Phys. Rev. A 98, 013835 (2018).

[42] A. Geraldi, S. De, A. Laneve, S. Barkhofen, J. Sperling, P. Mataloni, and C. Silberhorn, Subdiffusion via disordered quantum walks, Phys. Rev. Research 3, 023052 (2021).

[43] A. P. Schnyder, S. Ryu, A. Furusaki, and A. W. W. Ludwig, Classification of topological insulators and superconductors in three spatial dimensions, Phys. Rev. B 78, 195125 (2008).

[44] B. Tarasinski, J. K. Asbóth, and J. P. Dahlhaus, Scattering theory of topological phases in discrete-time quantum walks, Phys. Rev. A 89, 042327 (2014).

[45] C. Cedzich, T. Geib, F. A. Grünbaum, C. Stahl, L. Velázquez, A. H. Werner, and R. F. Werner, The topological classification of one-dimensional symmetric quantum walks, Ann. Henri Poincaré 19, 325 (2018).

[46] J. K. Asbóth, Symmetries, topological phases, and bound states in the one-dimensional quantum walk, Phys. Rev. B 86, 195414 (2012).

[47] P. W. Anderson, Absence of diffusion in certain random lattices, Phys. Rev. 109, 1492 (1958).

[48] F. Wegner, The mobility edge problem: Continuous symmetry and a conjecture, Z. Phys. B 35, 207 (1979).

[49] K. B. Efetov, A. I. Larkin, and D. E. Khmel'nitskii, Interaction of diffusion modes in the theory of localization, JETP 52, 568 (1980)

[50] A. M. Pruisken and L. Schäfer, The Anderson model for electron localisation non-linear $\sigma$ model, asymptotic gauge invariance, Nucl. Phys. B 200, 20 (1982).

[51] K. B. Efetov and A. I. Larkin, Kinetics of a quantum particle in long metallic wires, Sov. Phys. JETP 58, 444 (1983).

[52] K. B. Efetov, Sypersymmetry in Disorder and Chaos (Cambridge University Press, Cambridge, 1997).

[53] E. Abrahams, P. W. Anderson, D. C. Licciardello, and T. V. Ramakrishnan, Scaling Theory of Localization: Absence of Quantum Diffusion in Two Dimensions, Phys. Rev. Lett. 42, 673 (1979).

[54] I. C. Fulga, F. Hassler, A. R. Akhmerov, and C. W. J. Beenakker, Scattering formula for the topological quantum number of a disordered multimode wire, Phys. Rev. B 83, 155429 (2011).

[55] A. Altland, D. Bagrets, L. Fritz, A. Kamenev, and H. Schmiedt, Quantum Criticality of Quasi-One-Dimensional Topological Anderson Insulators, Phys. Rev. Lett. 112, 206602 (2014).

[56] Notice that this implies that $\sum_{q, \sigma^{\prime} \sigma} P_{\sigma^{\prime} \sigma}^{\text {chiral }}(t, q)=1 /\left(4 \ln ^{2} t\right)$ as shown in Appendix B.

[57] Y. G. Sinai, The limiting behavior of a one-dimensional random walk in a random medium, Theory Probab. Appl. 27, 256 (1982).

[58] J. Bouchaud, A. Comtet, A. Georges, and P. L. Doussal, Classical diffusion of a particle in a one-dimensional random force field, Ann. Phys. 201, 285 (1990). 
[59] A. Comtet and D. S. Dean, Exact results on Sinai's diffusion, J. Phys. A: Math. Gen. 31, 8595 (1998).

[60] Sinai diffusion has recently been suggested to leave traces of unconventional heat propagation in the form of nonmonotonically propagating thermal current pulses in quasi-onedimensional topological superconducting wires near criticality [14].

[61] J. K. Asbóth and H. Obuse, Bulk-boundary correspondence for chiral symmetric quantum walks, Phys. Rev. B 88, 121406(R) (2013).

[62] I. Mondragon-Shem, T. L. Hughes, J. Song, and E. Prodan, Topological Criticality in the Chiral-Symmetric AIII Class at Strong Disorder, Phys. Rev. Lett. 113, 046802 (2014).

[63] Topological boundary modes can be introduced, for example, by connecting two one-dimensional quantum walk systems characterized by $\hat{U}(\varphi=0, \theta)$ and $\hat{U}(\varphi=0,-\theta)$, respectively. The spin expectation of boundary modes are eigenvalues of chiral operator $\sigma_{2}$.

[64] Q. Zhao and J. Gong, From disordered quantum walk to physics of off-diagonal disorder, Phys. Rev. B 92, 214205 (2015).

[65] J. Boutari, A. Feizpour, S. Barz, C. D. Franco, M. S. Kim, W. S. Kolthammer, and I. A. Walmsley, Large scale quantum walks by means of optical fiber cavities, J. Opt. 18, 094007 (2016).

[66] X.-Y. Xu, Q.-Q. Wang, W.-W. Pan, K. Sun, J.-S. Xu, G. Chen, J.-S. Tang, M. Gong, Y.-J. Han, C.-F. Li, and G.-C. Guo, Measuring the Winding Number in a Large-Scale Chiral Quantum Walk, Phys. Rev. Lett. 120, 260501 (2018).

[67] T. Nitsche, S. Barkhofen, R. Kruse, L. Sansoni, M. Štefaňák, A. Gábris, V. Potoček, T. Kiss, I. Jex, and C. Silberhorn, Probing measurement-induced effects in quantum walks via recurrence, Sci. Adv. 4, eaar6444 (2018).

[68] B. Do, M. L. Stohler, S. Balasubramanian, D. S. Elliott, C. Eash, E. Fischbach, M. A. Fischbach, A. Mills, and B. Zwickl, Experimental realization of a quantum quincunx by use of linear optical elements, J. Opt. Soc. Am. B 22, 499 (2005).
[69] P. Xue, R. Zhang, H. Qin, X. Zhan, Z. H. Bian, J. Li, and B. C. Sanders, Experimental Quantum-Walk Revival with a Time-Dependent Coin, Phys. Rev. Lett. 114, 140502 (2015).

[70] H. Wang, J. Qin, X. Ding, M.-C. Chen, S. Chen, X. You, Y.-M. He, X. Jiang, L. You, Z. Wang, C. Schneider, J. J. Renema, S. Höfling, C.-Y. Lu, and J.-W. Pan, Boson Sampling with 20 Input Photons and a 60-Mode Interferometer in a $10^{14}$-Dimensional Hilbert Space, Phys. Rev. Lett. 123, 250503 (2019).

[71] Y. He, X. Ding, Z.-E. Su, H.-L. Huang, J. Qin, C. Wang, S. Unsleber, C. Chen, H. Wang, Y.-M. He, X.-L. Wang, W.-J. Zhang, S.-J. Chen, C. Schneider, M. Kamp, L.-X. You, Z. Wang, S. Höfling, C.-Y. Lu, and J.-W. Pan, Time-Bin-Encoded Boson Sampling with a Single-Photon Device, Phys. Rev. Lett. 118, 190501 (2017).

[72] Z. L. Yuan, B. Fröhlich, M. Lucamarini, G. L. Roberts, J. F. Dynes, and A. J. Shields, Directly Phase-Modulated Light Source, Phys. Rev. X 6, 031044 (2016).

[73] C. S. Hamilton, S. Barkhofen, L. Sansoni, I. Jex, and C. Silberhorn, Driven discrete time quantum walks, New J. Phys. 18, 073008 (2016).

[74] M. R. Zirnbauer, Supersymmetry for systems with unitary disorder: Circular ensembles, J. Phys. A: Math. Gen. 29, 7113 (1996).

[75] A. Altland, S. Gnutzmann, F. Haake, and T. Micklitz, A review of sigma models for quantum chaotic dynamics, Rep. Prog. Phys. 78, 086001 (2015).

[76] A. Lamacraft, B. D. Simons, and M. R. Zirnbauer, Localization from $\sigma$-model geodesics, Phys. Rev. B 70, 075412 (2004).

[77] A. Altland and R. Merkt, Spectral and transport properties of quantum wires with bond disorder, Nucl. Phys. B 607, 511 (2001).

[78] E. J. Meier, F. A. An, A. Dauphin, M. Maffei, P. Massignan, T. L. Hughes, and B. Gadway, Observation of the topological Anderson insulator in disordered atomic wires, Science 362, 929 (2018). 